\title{
IS THE ORDOS BLOGK ARCHEAN OR PALEOPROTEROZOIC IN AGE? IMPLICATIONS FOR THE PRECAMBRIAN EVOLUTION OF THE NORTH CHINA GRATON
}

\author{
YUSHENG WAN***,§§§ ${ }^{\dagger}$, HANGQIANG XIE****, HUA YANG***, ZEJIU WANG ${ }^{\S}$, \\ DUNYI LIU****, ALFRED KRÖNER****, SIMON A. WILDE*,\$ \\ YUANSHENG GENG*, LIUYI SUN***, MINGZHU MA***, SHOUJIE LIU***, \\ CHUNYAN DONG****, and LILIN DU*
}

\begin{abstract}
The Ordos Block is a large but poorly exposed crustal segment in the western part of the North China Craton. Little is known about its Precambrian basement rocks and their evolution because of an extensive cover by younger sedimentary rocks. We report SHRIMP zircon ages and Hf-in-zircon isotopic compositions for five samples recovered from drill holes that penetrated into the Ordos basement. Based on these data, two age groups of rocks can be distinguished: an early Paleoproterozoic group that is represented by one sample from the northern margin of the Ordos Block and a late (or middle-late) Paleoproterozoic group consisting of the remaining four samples. The early Paleoproterozoic sample contains zircon cores and metamorphic rims with ages of $>2.4 \mathrm{Ga}$ and $>2.28 \mathrm{Ga}$, respectively. The cores have $\varepsilon_{H f}(t)$ values of -8.8 to 6.2 and $H$ crustal model ages of 2606 to $3221 \mathrm{Ma}$. The remaining rocks of late (or middle-late) Paleoproterozoic age contain zircons that may also show a core-rim structure, but with the cores having ages of $\sim 2.08 \mathrm{Ga}$ and the metamorphic rims recording ages of $\sim 1.9 \mathrm{Ga}$. The zircon cores have $\varepsilon_{\mathrm{Hf}}(t)$ values of -9.1 to 10.1, and Hf crustal model ages of 2039 to $3068 \mathrm{Ma}$. Combined with data from earlier studies, we draw the conclusion that late Paleoproterozoic metasedimentary rocks are widespread in the Ordos basement. There is no evidence from our samples of an Archean component, and this calls into question the prevailing view that the Ordos basement is an Archean cratonic block. The fact that the Ordos basement was involved in a widespread late Paleoproterozoic tectono-thermal event indicates that most current models for the tectonic evolution of the western portion of the North China Craton need to be revised.
\end{abstract}

Key words: Ordos basement, early Precambrian, North China Craton, zircon dating, Hf analysis

\section{INTRODUCTION}

The North China Craton (NCC) is one of the largest cratonic blocks in the eastern Eurasian continent. It is generally accepted that the basement of the NCC is composed of several different blocks, separated by major tectonic boundaries, and that they were originally independent crustal entities that eventually collided to form the craton. However, there is still considerable debate as to how the craton should be subdivided, where the boundaries are located, and what was the timing of collision and amalgamation (Wu and others, 1998; Wu and Zhong 1998; Zhang and others, 1998; Zhao and others, 1998, 2001; Zhai and others, 2000, 2005; Zhai and Liu, 2003; Kusky and Li, 2003; Zheng and others, 2013). The number of blocks proposed in the various models ranges from 15 (Zhang and others, 1998) to 5 (Wu and others, 1998), but with little explanation of the nature of their boundaries.

* Institute of Geology, Chinese Academy of Geological Sciences, 26 Baiwanzhuang Road, Beijing 100037, China

** Beijing SHRIMP Center, 26 Baiwanzhuang Road, Beijing 100037, China

*** Changqing Oilfield Company of PetroChina, Xi'an 710018, China

$\$$ Chinese Academy of Geological Sciences, 26 Baiwanzhuang Road, Beijing 100037, China

$\$ \S$ Department of Applied Geology, Curtin University, Perth, WA 6845, Australia

sss State Key Laboratory for Continental Tectonics and Dynamics, Institute of Mineral Resources, Chinese Academy of Geological Sciences, Beijing 100037, China

${ }^{\dagger}$ Corresponding author: wanyusheng@bjshrimp.cn (Y. Wan) 


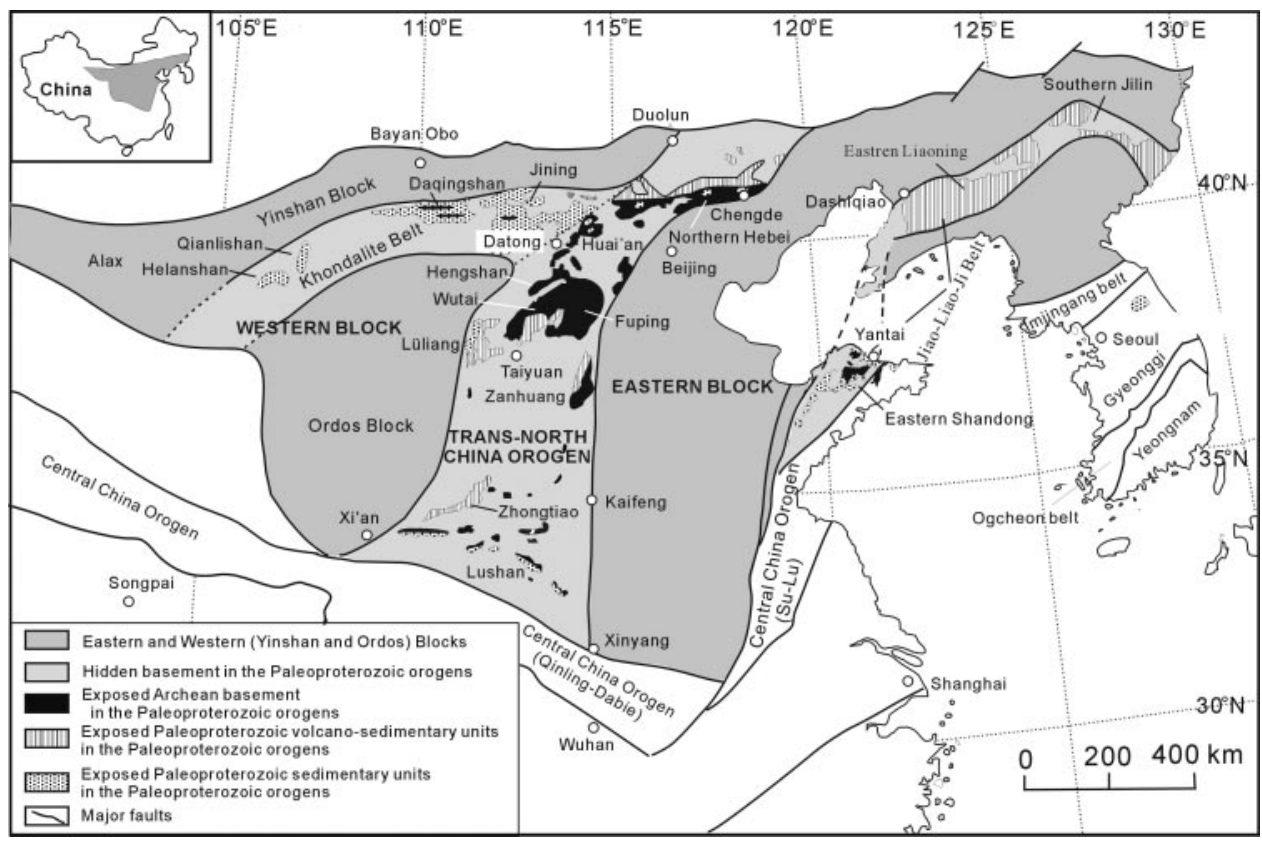

Fig. 1. Tectonic map of the North China Craton showing Paleoproterozoic orogenic belts. Modified after Zhao and others (2005), and Wan and others (2006).

There has been wide acceptance of the model proposed by Zhao and others (1998, 1999a, 1999b, 2001, 2005) who initially suggested a 3-fold subdivision of the craton, based on lithological, structural, metamorphic and geochronological differences (Zhao and others, 1998, 1999a, 1999b). These authors pointed out that metamorphic $\mathrm{P} / \mathrm{T}$ paths of widespread supracrustal assemblages were anticlockwise in the eastern part of the craton, clockwise in its central part, but changing from clockwise to anticlockwise in the western part (Zhao and others, 1999a, 1999b). They defined the central zone as the Trans-North China Orogen (TNCO) that separated the NCC into Eastern and Western Blocks. The late Archean anticlockwise P-T paths in the two blocks were interpreted to be due to several mantle plumes, resulting in magmatic underplating and high-grade metamorphism at $\sim 2.5 \mathrm{Ga}$ (Zhao and others, 1999a), whereas the clockwise paths in the TNCO was interpreted to reflect continental collision between the Eastern and Western Blocks, leading to Paleoproterozoic assembly of the NCC at $\sim 1.85 \mathrm{Ga}$ (Zhao and others, 2001). This model was later modified (Zhao and others, 2005) into the currently-favored 6-fold (or 7-fold) subdivision of the craton (fig. 1) (see also Wilde and others, 2002, 2004; Guo and others, 2005; Kröner and others, 2005, 2006; Wan and others, 2006, 2009). This modification resulted from the re-interpretation of major collisional zones within both the Eastern and Western Blocks. In the Eastern Block, rocks of the Jiao-Liao-Ji Belt were considered to have been deposited in a rift setting (Yang and others, 1988), and the rift was thought to have closed at $\sim 1.85 \mathrm{Ga}$, although other workers have favored either continent-arc collision (Bai 1993; Faure and others, 2004; Luo and others, 2004) or continent-continent collision (He and Ye, 1998) without prior rifting. In the Western Block, the east-west trending Khondalite Belt (also described as the Inner Mongolia Suture Zone, Santosh, 2010) separates the block into two component parts (Zhao and others, 2005): the Yinshan Block in the north and the Ordos Block in the south (fig. 1). 
In contrast to Zhao and several authors cited above, Li and others (2002), Kusky and $\mathrm{Li}$ (2003) and Kusky and others (2007) suggested that collision between the Eastern and Western Blocks occurred along a Central Zone in the Neoarchean $(\sim 2.5$ Ga) to form a unified NCC. Zhai and Santosh (2011) divided the NCG into six micro-blocks with very different boundaries from those proposed by Zhao and co-workers. They suggested that these micro-blocks were welded together along Neoarchean granite-greenstone belts at $\sim 2.5 \mathrm{Ga}$.

It is in the context of this debate that we report SHRIMP U-Pb and LA-ICP-MS Hf isotope analyses of zircons from rocks recovered from drill cores that penetrate basement beneath the Ordos Basin. These data show that the Ordos Block, as defined above, was involved in a late Paleoproterozoic tectono-thermal event, thus bringing into question whether the block is really an Archean cratonic fragment.

GEOLOGICAL BACKGROUND

The Yinshan Block forms the northern part of the Western Block of the NCG and is composed of TTG gneisses and ultramafic to felsic volcanic rocks, metamorphosed from greenschist to granulite facies. The high-grade TTG gneisses are associated with mafic granulites and charnockites, whereas the lower grade volcanic assemblages form linear belts associated with low-grade TTG suites (see Zhao and others, 1999b and references therein). Metamorphism in all domains is characterized by anti-clockwise P-T paths (Zhao and others, 1999b) and occurred at $\sim 2.5 \mathrm{Ga}$.

The Khondalite Belt to the south of the Yinshan Block is composed of khondalites (garnet-sillimanite metapelites), interlayered with charnockites, ortho- and paragneisses and various magmatic suites, all at upper amphibolite or granulite facies. The rocks were interpreted to have formed along the passive northern margin of the Ordos Block (see below), with subduction to the north below the Yinshan Block (Zhao and others, 2005). All metamorphic assemblages define tight clockwise $\mathrm{P} / \mathrm{T}$ paths (Zhao and others, 1999b and references therein). It appears that there were two distinct metamorphic events at the end of the Paleoproterozoic, namely an early high-grade event at $1.95 \mathrm{Ga}$ that was interpreted to reflect the collision of the Ordos and Yinshan Blocks (Zhao and others, 2010) and a slightly later, more local, UHT event at $1.92 \mathrm{Ga}$, interpreted as either due to massive intrusion of mafic magmatic rocks (Guo and others, 2011) or due to magmatic underplating or subduction-collision processes (Santosh and others, 2006, 2007; Tsunogae and others, 2011; Santosh and others, 2013). However, metamorphic zircon ages ranging almost continuously from 1.95 to 1.83 Ga have been identified in the Daqingshan area (Wan and others, 2013).

The Ordos Block makes up the southern part of the Western Block (figs. 1 and 2) and is the least understood tectonic unit in the NCC, because the Precambrian crystalline rocks are entirely covered by younger sedimentary rocks of the Ordos Basin. The block occupies an area of $250,000 \mathrm{~km}^{2}$, and its lithospheric structure is significantly different from that of the eastern NCC. Whereas craton destruction since the Mesozoic has resulted in significant lithospheric thinning $(60-100 \mathrm{~km})$ in the eastern NCC (Zhang and others, 2013, and references therein), the Ordos Block has remained a stable cratonic area with a thick lithosphere $(>200 \mathrm{~km})$, low heat flow, high-velocity anomalies, an absence of earthquakes, and an apparent lack of internal deformation since the late Paleoproterozoic (Wang and others, 1996; Hu and others, 2000; Zhai and Liu, 2003; Chen and others, 2009; Chen, 2010; Jiang and others, 2013). It is overlain by a Mesoproterozoic to Cenozoic sedimentary cover of great thickness $(>6000 \mathrm{~m})$, although Neoproterozoic to early Carboniferous strata are missing (COCP, 2011). Dating of detrital zircons from young sedimentary cover rocks and modern river sands that extend across the Ordos Block reveal evidence of tectono-thermal events of late Neoarchean and late Paleoproterozoic ages in their source regions (Song and others, 2010; Diwu and others, 2011; Wang and others, 2012; Xie and Heller, 2013). However, 


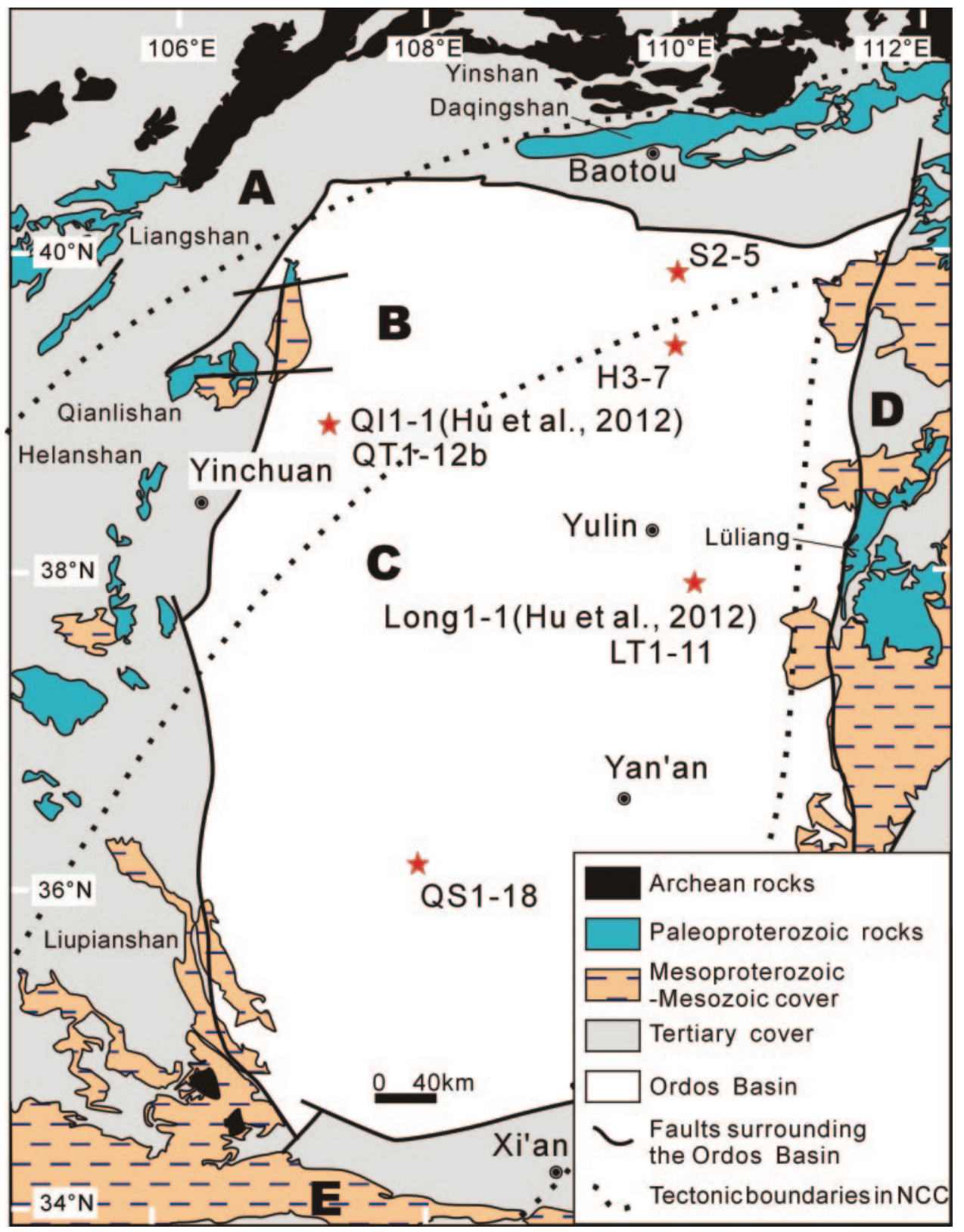

Fig. 2. Geological map of the Ordos Basin and surrounding areas (COCP, 2011), showing sample locations in this study and those of $\mathrm{Hu}$ and others (2012). A: Yinshan Block; B: Khondalite Belt; C: Ordos Block; D: Trans-North China Orogen; E: Qinling Orogen.

this does not imply that the Ordos basement (the term is used here for all crystalline rocks underlying the unmetamorphosed Ordos Basin sediments) underwent these events, because the Ordos Block itself was a depositional area, at least during the late Paleoproterozoic. 
Based on drill core data, the basement is composed of granulite, para- and orthogneiss, leptite (a fine-grained metamorphic rock with a granoblastic texture, mainly composed of feldspar and quartz), amphibolite, quartzite, schist and marble. It is divided into granulite, amphibolite and greenschist facies sub-areas, with the granulite facies rocks mainly occurring in the northern part (COCP, 2011). Hu and others (2012) carried out SHRIMP U-Pb zircon dating on two drill-core samples from holes drilled into Precambrian basement in the northwestern and eastern parts of the Ordos Basin (fig. 2). They concluded that the Ordos Block experienced strong magmatism during the late Paleoproterozoic and that there may be a Paleoproterozoic mobile belt in the region.

Precambrian rocks occur widely around the Ordos Basin. In the Lüliang area (fig. 2), east of the basin, the metamorphic basement is composed of the Jiehekou, Lüliang, and Yejishan "Groups"1 and various granitoids. Rocks of the Jiehekou "Group" extend in an east-west direction, and their pelitic protoliths are similar to the khondalites, although the rocks exhibit a lower-grade of metamorphism (commonly amphibolite facies). The Jiehekou "Group" is considered to have been deposited during the late Paleoproterozoic for the following reasons: 1) the youngest detrital zircons from metasedimentary rocks are $\sim 2.0 \mathrm{Ga}$ in age, and 2) the rocks underwent amphibolite facies metamorphism, but no strong metamorphism occurred after the late Paleoproterozoic in this area (Wan and others, 2006; Xia and others, 2009). The Lüliang and Yejishan "Groups" extend in a north-south direction and are composed of metamorphosed volcano-sedimentary rocks and record both magmatic and metamorphic zircon ages of $\sim 2.21 \mathrm{Ga}$ and $1.83 \mathrm{Ga}$ (Liu and others, 2011). Granitoid magmatism occurred at $2.50,2.37,2.18$ to $2.17,2.06$ and 1.83 to $1.79 \mathrm{Ga}$, with some granitoids recording metamorphic zircon ages of 1.87 to $1.84 \mathrm{Ga}$ (Geng and others, 2000, 2004, 2006; Liu and others, 2009; Zhao and others, 2008a).

In the Daqingshan area, north of the Ordos Basin (fig. 2), high-grade rocks, including granulites (the Sanggan or Xinghe "Group"), gneisses (the lower Wulashan "Group"), khondalites (the upper Wulashan "Group") and plutonic rocks, are widely distributed and extend in a general east-west direction. The khondalites were considered to be Archean in early studies, mainly based on their high metamorphic grade (Shen and others, 1990; Hu and others, 1994; Lu and others, 1996; Yang and others, 2000). However, these metasedimentary rocks contain detrital and metamorphic zircons with ages of $\sim 2.0$ and 1.95 to $1.83 \mathrm{Ga}$, respectively, suggesting deposition during the Paleoproterozoic (Wan and others, 2006; Zhao and others, 2010; Dong and others, 2012). Recent studies revealed multiple tectono-thermal events during the late Neoarchean to late Paleoproterozoic at 2.60 to $2.50,2.45$ to 2.37, 2.30 to 2.00 and 1.95 to $1.85 \mathrm{Ga}$, as suggested by SHRIMP U-Pb dating of magmatic, detrital and metamorphic zircons (Wan and others, 2006, 2008, 2009; Wu and others, 2006; Santosh and others, 2007, 2009; Zhong and others, 2007; Zhao and others, 2010; Li and others, 2010; Liu and others, 2012; Ma and others, 2012a, 2012b; Dong and others, 2012, 2013). Importantly, the earliest known Paleoproterozoic (2.50-2.45 Ga) supracrustal rocks in the NCG were identified in the Daqingshan area and underwent high-grade metamorphism between 2.45 and 2.40 Ga (Wan and others, 2009; Dong and others, 2013).

In the Qianlishan-Helanshan area, west of the Ordos Basin (fig. 2), the Precambrian basement includes rocks of the Qianlishan and Helanshan "Groups," as well as

\footnotetext{
${ }^{1}$ Strong deformation and metamorphism have overprinted most primary depositional features, and it is therefore incorrect to refer to these rocks as lithostratigraphic groups. Rather than using a different nomenclature, we retain the term "Group" in quotation marks and retain the names, since this will enable our work to be compared to the earlier Chinese literature.
} 
granitoids, mostly extending in an east-west direction. The supracrustal rocks are virtually identical in field appearance and formation ages to khondalites in the Daqingshan-Jining area (Dong and others, 2007; Yin and others, 2009; Zhou and Geng, 2009).

\section{SAMPLE SELECTION AND PETROGRAPHY}

Because the Ordos Block is critical to all models concerning the nature and evolution of the NCC, it is important that direct evidence as to the types of rocks present be obtained. Certain holes drilled to assess the petroleum potential of the Ordos Basin extend for up to one or several tens of meters into the basement rocks, and these provide the only direct evidence as to the nature and characteristics of the Ordos basement. Samples were obtained by Petrochina, and the drill cores are the subject of this research. Locations are shown in figure 2, and general information about the samples is provided in table 1 . Note that the samples are spread widely across the Ordos Basin, thus allowing us to evaluate the nature of the basement over a considerable area.

\section{Biotite K-Feldspar Gneiss (S2-5)}

This sample comes from a drill core obtained near the northern margin of the Ordos Basin (fig. 2). The medium-grained rock is dark gray in color and has a gneissic structure (fig. 3A). It is composed of quartz (30-40\%), K-feldspar (20-25\%), biotite $(10-20 \%)$, plagioclase $(8-10 \%)$ and minor secondary epidote $(3-5 \%)$. Biotite flakes and feldspar grains are oriented, with elongated quartz aggregates displaying undulose extinction and dynamic recrystallization. Some K-feldspar occurs as megacrysts. Some grains of plagioclase are sericiticized and saussuritized. Biotite is brownish-green and occurs as relatively small, oriented flakes along the boundaries of feldspar and quartz. Fine-grained opaque minerals are distributed along the cleavage of biotite. It is uncertain from the hand sample whether the rock is an ortho- or paragneiss.

\section{Banded Two-Mica K-Feldspar Gneiss (H3-7)}

This sample is from a drill core $\sim 50 \mathrm{~km}$ south of sample S2-5 (fig. 2). The rock is not uniform and is reddish to gray in color and shows a banded structure with some anatectic veins (fig. 3B). The overall mineral assemblage is quartz (35-40\%), K-feldspar $(20-25 \%)$, plagioclase $(3-5 \%)$, biotite $(3-5 \%)$, muscovite $(5-10 \%)$, and opaque minerals (mainly magnetite, $c a$. $10 \%$ ), with secondary epidote $(3-5 \%)$. The light layers are rich in quartz and feldspar with minor biotite and magnetite. Quartz shows weak undulose extinction. Gray layers are dominated by biotite, muscovite and magnetite. Some small opaque grains (probably ilmenite) occur as exsolution lamellae along the cleavage of muscovite and biotite flakes. Again, it is uncertain from the hand sample whether the rock is an ortho- or paragneiss.

\section{Garnet-Sillimanite-Biotite Gneiss (QT1-12b)}

Our sample, QT1-12b, comes from the same drill core from which $\mathrm{Hu}$ and others (2012) obtained their sample QI1-1 and is located in the northwestern part of the Ordos Basin, about $100 \mathrm{~km}$ northeast of Yinchuan (fig. 2). Sample QI1-1, from a depth of $5135 \mathrm{~m}$, is composed of plagioclase $(40 \%)$, quartz $(35 \%)$, sillimanite $(10 \%)$, K-feldspar (5\%), garnet (5\%) and biotite (5\%) (Hu and others, 2012). Our sample QT1-12b was taken from a depth of 5229 to $5233 \mathrm{~m}$ and is red to gray in color, coarse-grained, and has a gneissic structure (fig. 3C). Overall, the rock is composed of quartz (20-30\%), K-feldspar (15-20\%), biotite (10-15\%), garnet (5-10\%), sillimanite $(10-15 \%)$, plagioclase $(3-5 \%)$ and opaque minerals $(3-5 \%)$. The opaque minerals are magnetite/ilmenite, with minor graphite. The foliation is defined by oriented sillimanite and biotite, whereas quartz shows strong dynamic recrystallization, indicating 


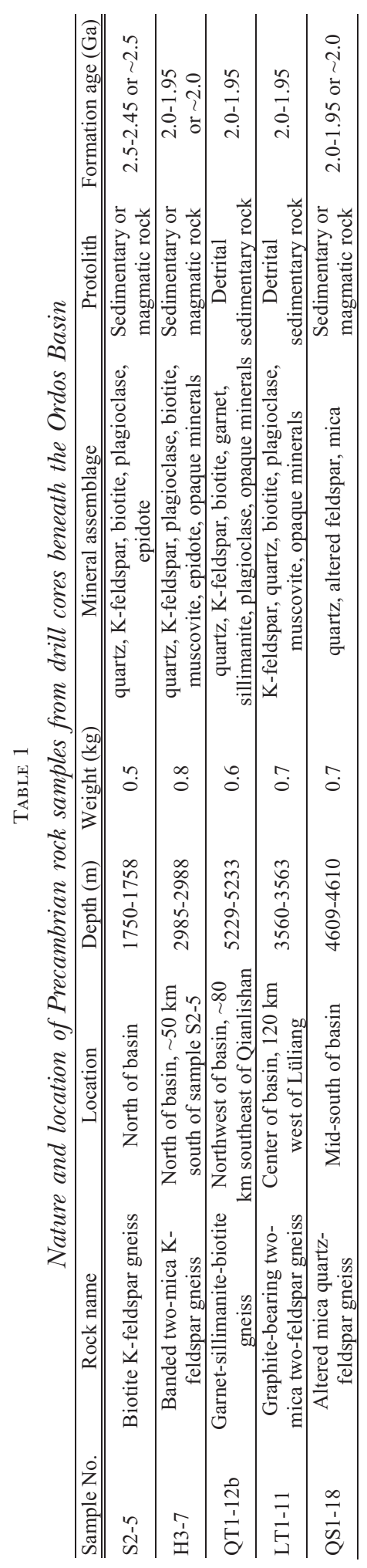



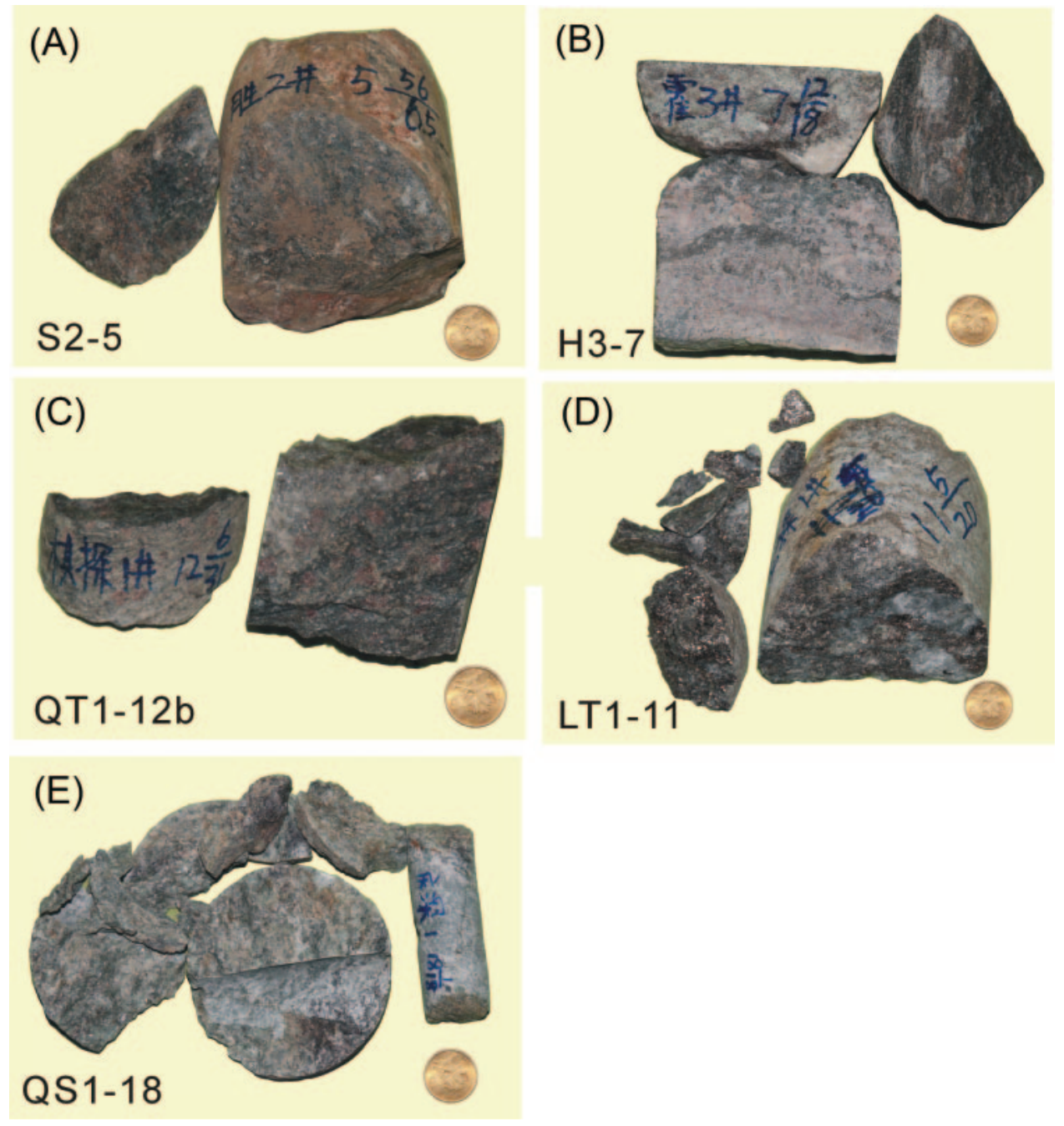

Fig. 3. Photographs of Paleoproterozoic rocks obtained from drill cores beneath the Ordos Basin. (A) Biotite K-feldspar gneiss (S2-5); (B) banded two-mica K-feldspar gneiss (H3-7); (C) garnet-sillimanitebiotite gneiss (QT1-12b); (D) graphite-bearing two-mica two-feldspar gneiss (LT1-11); (E) altered mica quartz-feldspar gneiss (QS1-18). The coin is $2 \mathrm{~cm}$ in diameter.

ductile deformation. Garnet occurs as large porphyroblasts with quartz inclusions in the cores; some garnets show embayed margins. Needle-like sillimanite aggregates occur along with biotite, plagioclase and K-feldspar, and this assemblage indicates dehydration melting. K-feldspar is mainly perthite, supporting a high-temperature history for the gneiss. From the mineral assemblage, the rock is a paragneiss.

\section{Graphite-Bearing Two-Mica Two-Feldspar Gneiss (LT1-11)}

This sample was taken from a depth of 3560 to $3563 \mathrm{~m}$ in the east-central part of the Ordos Basin (fig. 2). The rock is medium- to coarse-grained and has a gneissic structure with coarse-grained feldspar and quartz aggregates occurring as veins (fig. 3D). It is composed of K-feldspar (30-35\%), quartz (20-30\%), biotite (10-15\%), 
plagioclase $(5-10 \%)$, muscovite $(5-10 \%)$ and opaque minerals $(2-3 \%)$ that are mainly graphite with jamesonite and magnetite. Most plagioclase is altered to sericite and forms irregular or subhedral grains with polysynthetic or combined albite and pericline twins. Some plagioclase grains exhibit myrmekitic textures along their margins. Biotite and muscovite are oriented together with minor graphite and other opaque minerals and delineate the foliation in the rock. Strong deformation is also supported by undulose extinction and dynamic recrystallization of most quartz grains. From the same drill core, but from a shallower depth $(3495 \mathrm{~m}), \mathrm{Hu}$ and others (2012) collected sample Long1-1. It is similar to our sample LT1-11, consisting of quartz $(35 \%)$, K-feldspar $(35 \%)$, plagioclase $(25 \%)$, biotite $(3 \%)$ and muscovite $(2 \%)$. The authors considered that their sample was a granitic gneiss that contains schist enclaves. Our interpretation is that both samples are most likely paragneisses that have experienced anatexis for reasons that: 1) sample LT1-11 contains graphite; 2) minerals show a heterogeneous distribution; 3) the "enclaves" are similar in lithology to the garnetsillimanite-biotite gneiss ( $\mathrm{Hu}$ and others, 2012) that contains detrital zircons with the same ages as those of zircons from the "host" gneiss.

\section{Altered Mica Quartz-Feldspar Gneiss (QS1-18)}

This sample was taken from a drill core in the south-central part of the Ordos Basin (fig. 2) and is a gray, coarse-grained rock with a gneissic structure (fig. 3E). It is mainly composed of quartz (50-55\%), altered feldspar (30-35\%) and muscovite $(5-10 \%)$. Quartz and altered feldspar grains reach up to $5 \mathrm{~mm}$ in diameter and show triple-point junctions, indicating structural equilibrium. Muscovite flakes reach $\sim 1$ $\mathrm{mm}$ in size and commonly occur together with altered feldspar. Exsolution of opaque minerals (hematite, martite and anatase) occurs along the cleavage of some muscovite flakes, probably suggesting that they are formed as a result of retrogression of biotite. The feldspar grains show strong sericitization, and may originally be plagioclase. Therefore, quartz + altered feldspar + muscovite cannot represent the original mineral association of the sample, and it is not muscovite granite, although it is still uncertain whether this rock is an ortho- or paragneiss.

Of the five samples described above, three are composed of common rockforming minerals (feldspar, quartz, and mica) and underwent strong metamorphism and anatexis (S2-5, H3-7, QS1-18), thus it is difficult to determine whether their protoliths are sediments or granitoid rocks. However, we favor a supracrustal origin for the following reasons: 1 ) the samples are not homogeneous and show large variations in mineral proportions, being different from anatectic orthogneisses; 2) the samples have major element contents that are different from those of common magmatic rocks: $\mathrm{S} 2-5: \mathrm{SiO}_{2}=67.04 \%, \mathrm{Na}_{2} \mathrm{O}=1.60 \%, \mathrm{~K}_{2} \mathrm{O}=7.48 \%$; QS1-18: $\mathrm{SiO}_{2}=72.95 \%$, $\mathrm{Na}_{2} \mathrm{O}=0.01 \%, \mathrm{~K}_{2} \mathrm{O}=4.76 \%$; and $\mathrm{H} 3-7: \mathrm{SiO}_{2}=64.45 \%$, FeOt $=5.95 \%, \mathrm{Na}_{2} \mathrm{O}=$ $1.75 \%, \mathrm{~K}_{2} \mathrm{O}=5.94 \%$ (Y. S. Geng, unpublished data). All samples are located in sediment fields in $100 * \mathrm{mg}-\mathrm{c}-(\mathrm{al}-\mathrm{alk}), \mathrm{c}-\mathrm{mg}$ and $[(\mathrm{al}+\mathrm{fm})-(\mathrm{c}+\mathrm{alk})]-\mathrm{si}$ diagrams (not shown), which are constructed based on Niggli values.

\section{ANALYTICAL TECHNIQUES}

Zircons were hand-picked from heavy mineral concentrates and mounted in epoxy resin. The mount was ground down and polished so that the zircon cores were exposed, and zircons were imaged using cathodoluminescence (CL) to determine the best locations for U-Pb and $\mathrm{Hf}$ analyses. The mount was then cleaned and gold-coated. CL imaging was carried out using a 305 Hitachi SEM S-3000N equipped with a Gatan ChromaCL detector and a DigiSan II data306 recorder at the Beijing SHRIMP Center, Chinese Academy of Geological Sciences (CAGS). Operating conditions were $9 \mathrm{kV}$ and $99 \mu \mathrm{A}$. 
Zircon dating was carried out using the SHRIMP II at the Beijing SHRIMP Center, CAGS. The analytical procedures and conditions were similar to those described by Williams (1998). The intensity of the primary $\mathrm{O}^{2-}$ ion beam was $3.5 \mathrm{nA}$, the spot size was $\sim 30 \mu \mathrm{m}$, and each analytical site was rastered for 2.5 minutes prior to analysis. Five scans through the mass stations were made for each age determination of metamorphic zircons, but three or four scans were made on detrital zircon cores. Reference zircon SL13, with a U content of 238 ppm (Williams, 1998), and TEMORA 1 with a ${ }^{206} \mathrm{~Pb} /{ }^{238} \mathrm{U}$ age of $417 \mathrm{Ma}$ (Black and others, 2003), were used for elemental abundance and calibration of ${ }^{206} \mathrm{~Pb} /{ }^{238} \mathrm{U}$, respectively. Common lead corrections were applied using the measured ${ }^{204} \mathrm{~Pb}$ abundances and the model $\mathrm{Pb}$ compositions of Cumming and Richards (1975). Data processing was carried out using the SQUID and ISOPLOT programs (Ludwig, 2001, 2003). Uncertainties in the isotopic ratios of individual analyses in table 2 and on the concordia diagrams are given at $1 \sigma$, whereas uncertainties for weighted mean ages in the text are quoted at the 95 percent confidence level.

Hf-in-zircon isotope analyses were carried out using a Newwave UP213 laserablation microprobe, attached to a Neptune multi-collector ICP-MS, at the Institute of Mineral Resources, CAGS. Instrumental conditions and data acquisition were described by Hou and others (2007). Beam diameter was $40 \mu \mathrm{m}$, and helium was used as the carrier gas to transport the ablated sample from the laser-ablation cell to the ICP-MS torch via a mixing chamber, where it was mixed with argon. In order to correct for isobaric interferences of ${ }^{176} \mathrm{Lu}$ and ${ }^{176} \mathrm{Yb}$ on ${ }^{176} \mathrm{Hf}$, ratios of ${ }^{176} \mathrm{Lu} /{ }^{175} \mathrm{Lu}=0.02658$ and ${ }^{176} \mathrm{Yb} /{ }^{173} \mathrm{Yb}=0.796218$ were determined (Chu and others, 2002). For instrumental mass bias correction, $\mathrm{Yb}$ isotope ratios were normalized to ${ }^{172} \mathrm{Yb} /{ }^{173} \mathrm{Yb}$ of 1.35274 (Chu and others, 2002) and Hf isotope ratios to ${ }^{179} \mathrm{Hf} /{ }^{177} \mathrm{Hf}$ of 0.7325 , using an exponential law. The mass bias behavior of $\mathrm{Lu}$ was assumed to follow that of $\mathrm{Yb}$, and mass bias correction protocols were as described in Hou and others (2007). Zircon GJ1 was used as a reference standard with a weighted mean ${ }^{176} \mathrm{Hf} /{ }^{177} \mathrm{Hf}$ ratio of $0.282012 \pm 0.000021$ $(2 \sigma, \mathrm{n}=12)$ during our analyses. This is indistinguishable from a weighted mean ${ }^{176} \mathrm{Hf} /{ }^{177} \mathrm{Hf}$ ratio of $0.282013 \pm 0.000019(2 \sigma)$ obtained from in-situ analysis by Elhlou and others (2006). Data processing used a decay constant for ${ }^{176} \mathrm{Lu}$ of $1.867 \times 10^{-11} \mathrm{a}^{-1}$ (Söderlund and others, 2004), and the present-day chondritic ratios of ${ }^{176} \mathrm{Hf} /{ }^{177} \mathrm{Hf}=$ 0.282785 and ${ }^{176} \mathrm{Lu} /{ }^{177} \mathrm{Hf}=0.0336$ (Bouvier and others, 2008) were adopted to calculate $\varepsilon_{\mathrm{Hf}}(\mathrm{t})$ values. Hf crustal model ages $\left(\mathrm{t}_{\mathrm{DM}}{ }^{\mathrm{c}}\right)$ were estimated by projecting the initial ${ }^{176} \mathrm{Hf} /{ }^{177} \mathrm{Hf}$ of the zircons back to the depleted mantle growth curve using a ${ }^{176} \mathrm{Lu} /{ }^{177} \mathrm{Hf}$ ratio of 0.015 for the average continental crust (Griffin and others, 2000).

\section{ZIRCON CHARACTERISTICS AND GEOCHRONOLOGY}

Although we favor sedimentary protoliths for all the samples, for samples S2-5, H3-7 and QS1-18, a magmatic origin cannot be completely excluded. In the following sections, therefore, the term "core" is only used in a descriptive sense for the central domains of zircons from these samples, with no genetic implication. We use the term "detrital core" for the central domains of zircons from samples QT1-1 and LT1-11, which are undoubtedly paragneisses.

\section{Biotite K-Feldspar Gneiss (S2-5)}

Zircons are either oval or long-prismatic with sharp or rounded terminations, and many show a core-rim structure in cathodoluminescence (CL) images (figs. 4A, 4B, and $4 \mathrm{C}$ ). The rounded grains commonly have wider rims than the elongate grains. The cores show oscillatory or banded zoning, and most underwent recrystallization to varying extents. Thirty analyses were undertaken on 27 zircon grains (table 2 ). Twenty 
TABLE 2

SHRIMP U-Pb data for zircons from Precambrian rocks obtained from drill cores beneath the Ordos Basin

\begin{tabular}{|c|c|c|c|c|c|c|c|c|c|c|c|c|c|c|c|}
\hline \multirow[t]{2}{*}{ Spot } & $\begin{array}{c}\mathrm{U} \\
(\mathrm{ppm})\end{array}$ & $\begin{array}{c}\text { Th } \\
\text { (ppm) }\end{array}$ & $\frac{\mathrm{Th}}{\mathrm{U}}$ & $\begin{array}{l}{ }^{206} \mathrm{~Pb}^{*} \\
\text { (ppm) }\end{array}$ & \multirow[t]{2}{*}{$\frac{207 \mathrm{~Pb}^{*}}{206 \mathrm{~Pb}^{*}}$} & $\pm \%$ & $\frac{207 \mathrm{~Pb}^{*}}{235 \mathrm{U}}$ & $\pm \%$ & $\frac{206 \mathrm{~Pb}^{*}}{238 \mathrm{U}}$ & $\pm \%$ & $\begin{array}{l}\text { err } \\
\text { corr }\end{array}$ & $\begin{array}{l}\frac{206 \mathrm{~Pb}}{238 \mathrm{U}} \\
\text { Age }\end{array}$ & $\begin{array}{r}\frac{207 \mathrm{~F}}{206 \mathrm{~F}} \\
\mathrm{Ag}\end{array}$ & & $\begin{array}{c}\text { Discordance } \\
(\%)\end{array}$ \\
\hline & -felds & Ir gneiss & $(\mathrm{S} 2-5$ & & & & & & & & & & & & \\
\hline $1.1 \mathrm{C}$ & 929 & 360 & 0.40 & 212 & 0.1292 & 1.4 & 4.6 & 2.6 & 0.2583 & 2.1 & 0.83 & $1481 \pm 28$ & 2087 & \pm 25 & 29 \\
\hline $2.1 \mathrm{C}$ & 318 & 225 & 0.73 & 80 & 0.1595 & 0.77 & 6.42 & 2.3 & 0.2922 & 2.2 & 0.94 & $1652 \pm 32$ & 2450 & \pm 13 & 33 \\
\hline 3.1RC & 241 & 50 & 0.21 & 66 & 0.1595 & 0.74 & 6.96 & 2.3 & 0.3163 & 2.2 & 0.95 & $1772 \pm 34$ & 2450 & \pm 12 & 28 \\
\hline $4.1 \mathrm{C}$ & 331 & 223 & 0.70 & 94 & 0.1453 & 1.00 & 6.52 & 2.4 & 0.3254 & 2.2 & 0.90 & $1816 \pm 35$ & 2291 & \pm 18 & 21 \\
\hline $5.1 \mathrm{C}$ & 874 & 307 & 0.36 & 67 & 0.1373 & 1.10 & 1.657 & 2.4 & 0.0876 & 2.2 & 0.88 & $541 \pm 11$ & 2193 & \pm 20 & 75 \\
\hline $5.2 \mathrm{C}$ & 407 & 235 & 0.60 & 93 & 0.1441 & 1.00 & 5.2 & 2.4 & 0.2619 & 2.2 & 0.91 & $1499 \pm 29$ & 2277 & \pm 18 & 34 \\
\hline $6.1 \mathrm{C}$ & 463 & 178 & 0.40 & 56 & 0.1355 & 1.40 & 2.592 & 2.8 & 0.1388 & 2.4 & 0.86 & $838 \pm 19$ & 2170 & \pm 25 & 61 \\
\hline 7.1C & 463 & 219 & 0.49 & 150 & 0.1610 & 0.53 & 8.33 & 2.2 & 0.3752 & 2.2 & 0.97 & $2054 \pm 38$ & 2466 & \pm 9 & 17 \\
\hline $7.2 \mathrm{R}$ & 1968 & 240 & 0.13 & 436 & 0.1405 & 0.19 & 5 & 2.5 & 0.2581 & 2.5 & 1.00 & $1480 \pm 33$ & 2233 & \pm 3 & 34 \\
\hline $8.1 \mathrm{C}$ & 577 & 289 & 0.52 & 156 & 0.1434 & 1.50 & 6.09 & 2.7 & 0.3083 & 2.2 & 0.82 & $1732 \pm 33$ & 2269 & \pm 26 & 24 \\
\hline $9.1 \mathrm{C}$ & 227 & 109 & 0.50 & 65 & 0.1593 & 0.81 & 7.21 & 2.3 & 0.3284 & 2.2 & 0.94 & $1830 \pm 35$ & 2448 & \pm 14 & 25 \\
\hline $10.1 \mathrm{C}$ & 519 & 276 & 0.55 & 123 & 0.1444 & 0.83 & 5.41 & 2.3 & 0.2719 & 2.1 & 0.93 & $1550 \pm 29$ & 2280 & \pm 14 & 32 \\
\hline 11.1RC & 237 & 93 & 0.40 & 70 & 0.1572 & 0.76 & 7.46 & 2.9 & 0.3439 & 2.8 & 0.97 & $1905 \pm 46$ & 2426 & \pm 13 & 21 \\
\hline $12.1 \mathrm{C}$ & 900 & 454 & 0.52 & 170 & 0.1363 & 0.75 & 4.082 & 2.3 & 0.2172 & 2.1 & 0.94 & $1267 \pm 25$ & 2181 & \pm 13 & 42 \\
\hline $12.2 \mathrm{R}$ & 1201 & 73 & 0.06 & 206 & 0.1433 & 0.23 & 3.94 & 2.7 & 0.1996 & 2.7 & 0.99 & $1173 \pm 29$ & 2268 & \pm 4 & 48 \\
\hline $13.1 \mathrm{C}$ & 323 & 86 & 0.28 & 92 & 0.1583 & 0.36 & 7.18 & 2.5 & 0.3290 & 2.5 & 0.99 & $1834 \pm 40$ & 2438 & \pm 6 & 25 \\
\hline $14.1 \mathrm{C}$ & 483 & 238 & 0.51 & 166 & 0.1552 & 0.25 & 8.54 & 2.6 & 0.3990 & 2.6 & 0.99 & $2165 \pm 48$ & 2404 & \pm 4 & 0 \\
\hline 16.1C & 229 & 197 & 0.89 & 58 & 0.1636 & 0.41 & 6.64 & 2.7 & 0.2943 & 2.7 & 0.99 & $1663 \pm 39$ & 2493 & \pm 7 & 3 \\
\hline $17.1 \mathrm{R}$ & 1637 & 82 & 0.05 & 251 & 0.1296 & 0.32 & 3.187 & 2.6 & 0.1784 & 2.6 & 0.99 & $1058 \pm 25$ & 2092 & \pm 6 & 9 \\
\hline $18.1 \mathrm{R}$ & 3918 & 98 & 0.03 & 561 & 0.1184 & 0.17 & 2.72 & 2.5 & 0.1666 & 2.5 & 1.00 & $993 \pm 23$ & 1933 & \pm 3 & 49 \\
\hline $19.1 \mathrm{C}$ & 3637 & 1643 & 0.47 & 184 & 0.1272 & 0.50 & 1.03 & 2.6 & 0.0588 & 2.6 & 0.98 & $368 \pm 9$ & 2059 & \pm 9 & 82 \\
\hline $20.1 \mathrm{C}$ & 635 & 548 & 0.89 & 146 & 0.1578 & 0.25 & 5.83 & 2.5 & 0.2678 & 2.5 & 0.99 & $1530 \pm 34$ & 2433 & \pm 4 & 37 \\
\hline $21.1 \mathrm{R}$ & 2214 & 286 & 0.13 & 217 & 0.1387 & 0.53 & 2.185 & 2.5 & 0.1142 & 2.5 & 0.98 & $697 \pm 16$ & 2211 & \pm 9 & 8 \\
\hline $22.1 \mathrm{C}$ & 517 & 471 & 0.94 & 141 & 0.1687 & 0.90 & 7.37 & 2.7 & 0.3170 & 2.5 & 0.94 & $1775 \pm 39$ & 2544 & \pm 15 & 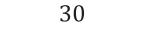 \\
\hline $23.1 \mathrm{R}$ & 1330 & 211 & 0.16 & 227 & 0.1485 & 0.33 & 4.06 & 2.5 & 0.1984 & 2.5 & 0.99 & $1167 \pm 27$ & 2329 & \pm 6 & 50 \\
\hline $24.1 \mathrm{C}$ & 559 & 307 & 0.57 & 139 & 0.1542 & 0.30 & 6.14 & 2.5 & 0.2887 & 2.5 & 0.99 & $1635 \pm 36$ & 2393 & \pm 5 & 32 \\
\hline $25.1 \mathrm{R}$ & 1060 & 56 & 0.05 & 300 & 0.1442 & 0.88 & 6.37 & 2.6 & 0.3206 & 2.5 & 0.96 & $1793 \pm 40$ & 2278 & \pm 13 & 21 \\
\hline $26.1 \mathrm{R}$ & 1899 & 275 & 0.15 & 221 & 0.1058 & 3.60 & 1.897 & 4.3 & 0.1302 & 2.5 & 0.59 & $789 \pm 19$ & 1727 & \pm 64 & 54 \\
\hline $27.1 \mathrm{C}$ & 325 & 190 & 0.61 & 56 & 0.1479 & 0.73 & 4.05 & 3.2 & 0.1984 & 3.1 & 0.97 & $1167 \pm 33$ & 2322 & \pm 12 & 50 \\
\hline $27.2 \mathrm{C}$ & 832 & 278 & 0.35 & 150 & 0.1302 & 1.30 & 3.66 & 2.8 & 0.2039 & 2.6 & 0.91 & $1196 \pm 28$ & 2099 & \pm 21 & 43 \\
\hline n & $0-m$ & X-fel & $\operatorname{ar}$ & $\sec ($ & 3-7) & & & & & & & & & & \\
\hline $1.1 \mathrm{C}$ & 416 & 256 & 0.64 & 58 & 0.1175 & 1.80 & 5 & 2.8 & 99 & 2.1 & 0.77 & $56 \quad \pm 19$ & 19 & \pm 32 & 50 \\
\hline $2.1 \mathrm{RC}$ & 281 & 69 & 0.26 & 75 & 0.1197 & 0.69 & 5.121 & 2.2 & 0.3102 & 2.1 & 0.95 & $1742 \pm 33$ & $1952=$ & \pm 12 & 11 \\
\hline $2.2 \mathrm{R}$ & 705 & 17 & 0.02 & 177 & 0.1146 & 0.79 & 4.602 & 2.3 & 0.2913 & 2.1 & 0.94 & $1648 \pm 31$ & 1874 & \pm 14 & 12 \\
\hline $3.1 \mathrm{C}$ & 264 & 401 & 1.57 & 40 & 0.1145 & 1.60 & 2.761 & 2.8 & 0.1749 & 2.3 & 0.82 & $1039 \pm 22$ & 1872 & \pm 29 & 45 \\
\hline $3.2 \mathrm{RC}$ & 420 & 286 & 0.70 & 71 & 0.1138 & 1.10 & 3.053 & 2.4 & 0.1946 & 2.1 & 0.89 & $1146 \pm 22$ & 1861 & \pm 20 & 38 \\
\hline $4.1 \mathrm{C}$ & 189 & 160 & 0.87 & 57 & 0.1204 & 0.88 & 5.810 & 3.7 & 0.3500 & 3.6 & 0.97 & $1933 \pm 60$ & 1963 & \pm 16 & 2 \\
\hline $5.1 \mathrm{C}$ & 134 & 84 & 0.65 & 42 & 0.1231 & 0.88 & 6.132 & 2.7 & 0.3614 & 2.5 & 0.95 & $1989 \pm 43$ & 2001 & \pm 16 & 1 \\
\hline $5.2 \mathrm{R}$ & 1789 & 32 & 0.02 & 113 & 0.1075 & 2.10 & 1.049 & & 0.0708 & 2.1 & 0.71 & $441 \pm 9$ & 1757 & \pm 39 & 75 \\
\hline $6.1 \mathrm{C}$ & 311 & 188 & 0.62 & 80 & 0.1288 & 0.79 & 5.271 & 3.3 & 0.2968 & 3.2 & 0.97 & $1675 \pm 47$ & 2081 & \pm 14 & 20 \\
\hline $7.1 \mathrm{C}$ & 484 & 117 & 0.25 & 95 & 0.154 & 1.20 & 4.820 & 2.5 & 0.2270 & 2.2 & 0.88 & $1319 \pm 26$ & 2391 & \pm 21 & 45 \\
\hline $8.1 \mathrm{C}$ & 492 & 106 & 0.22 & 78 & 0.1217 & 0.87 & 3.074 & 2.6 & 0.1832 & 2.4 & 0.94 & $1084 \pm 24$ & 1982 & \pm 15 & 45 \\
\hline $8.2 \mathrm{R}$ & 2511 & 40 & 0.02 & 94 & 0.088 & 2.70 & 0.512 & 3.4 & 0.04225 & 2.1 & 0.62 & $267 \pm 6$ & 1382 & \pm 52 & 81 \\
\hline $10.1 \mathrm{C}$ & 310 & 375 & 1.25 & 42 & 0.1109 & 2.10 & 2.355 & & 0.1540 & 2.2 & 0.73 & $923 \pm 19$ & 1815 & \pm 38 & 49 \\
\hline $11.1 \mathrm{R}$ & 982 & 128 & 0.13 & 712 & 0.1042 & 1.70 & 1.193 & 3.1 & 0.0830 & 2.6 & 0.83 & $514 \pm 13$ & 1700 & \pm 32 & 70 \\
\hline $11.2 \mathrm{RC}$ & 269 & 117 & 0.45 & 54 & 0.1186 & 3.30 & 3.751 & 4.3 & 0.2296 & 2.7 & 0.64 & $1332 \pm 33$ & 1935 & \pm 59 & 31 \\
\hline $12.1 \mathrm{R}$ & 1249 & 228 & 0.19 & 93 & 0.1001 & 2.40 & 1.150 & 3.2 & 0.0834 & 2.2 & 0.66 & $516 \pm 11$ & 1626 & \pm 45 & 68 \\
\hline $13.1 \mathrm{C}$ & 215 & 131 & 0.63 & 49 & 0.1179 & 1.50 & 4.201 & 2.7 & 0.2584 & 2.2 & 0.83 & $1482 \pm 29$ & 1925 & \pm 26 & 23 \\
\hline $14.1 \mathrm{C}$ & 47 & 27 & 0.59 & 12 & 0.1177 & 2.20 & 4.880 & 3.3 & 0.3009 & 2.4 & 0.74 & $1696 \pm 36$ & 1922 & \pm 40 & 12 \\
\hline $14.2 \mathrm{R}$ & 2004 & 658 & 0.34 & 99 & 0.1004 & 2.00 & 0.777 & 2.9 & 0.0561 & 2.2 & 0.74 & $352 \pm 7$ & 1631 & \pm 37 & 78 \\
\hline $15.1 \mathrm{C}$ & 158 & 99 & 0.65 & 49 & 0.1272 & 1.00 & 6.280 & 2.5 & 0.3583 & 2.2 & 0.91 & $1974 \pm 38$ & 2059 & \pm 18 & 4 \\
\hline 16.1RC & 389 & 124 & 0.33 & 100 & 0.1169 & 0.70 & 4.821 & 2.4 & 0.2990 & 2.3 & 0.96 & $1686 \pm 34$ & 1909 & \pm 13 & 12 \\
\hline Garnet- & $\operatorname{man}$ & biotit & gneis & s (QT1- & $12 \mathrm{~b})$ & & & & & & & & & & \\
\hline 1.1D & 93 & 45 & 0.49 & 29 & 0.1232 & 1.30 & 6.014 & 2.6 & 0.3539 & 2.3 & 0.86 & $1953 \pm 38$ & 2003 & \pm 24 & 2 \\
\hline 1.2RD & 368 & 89 & 0.25 & 109 & 0.1256 & 0.68 & 5.983 & 2.3 & 0.3452 & 2.2 & 0.96 & $1912 \pm 37$ & 2038 & \pm 12 & 6 \\
\hline 2.1D & 363 & 359 & 1.02 & 97 & 0.1263 & 0.76 & 5.432 & 2.5 & 0.3117 & 2.4 & 0.95 & $1749 \pm 36$ & 2048 & \pm 13 & 15 \\
\hline $3.1 \mathrm{R}+\mathrm{D}$ & 325 & 113 & 0.36 & 55 & 0.1182 & 0.84 & 3.199 & 2.3 & 0.1963 & 2.2 & 0.93 & $1155 \pm 23$ & 1929 & \pm 15 & 40 \\
\hline 3.2D & 382 & 163 & 0.44 & 86 & 0.1189 & 0.75 & 4.277 & 2.3 & 0.2608 & 2.2 & 0.95 & $1494 \pm 29$ & 1940 & \pm 13 & 23 \\
\hline $4.1 \mathrm{R}$ & 479 & 125 & 0.27 & 138 & 0.1176 & 0.79 & 5.441 & 2.3 & 0.3357 & 2.1 & 0.94 & $1866 \pm 35$ & 1920 & \pm 14 & 3 \\
\hline $2 \mathrm{D}$ & 321 & 81 & 0.26 & 105 & 0.131 & 81 & 6.913 & 2.3 & 0.3814 & 2.2 & 0.94 & $2083 \pm 39$ & 2118 & \pm 14 & 2 \\
\hline
\end{tabular}


TABle 2

(continued)

\begin{tabular}{|c|c|c|c|c|c|c|c|c|c|c|c|c|c|c|c|}
\hline Spot & $\begin{array}{c}\mathrm{U} \\
(\mathrm{ppm})\end{array}$ & $\begin{array}{c}\text { Th } \\
(\mathrm{ppm})\end{array}$ & $\frac{\mathrm{Th}}{\mathrm{U}}$ & $\begin{array}{l}206 \mathrm{~Pb}^{*} \\
(\mathrm{ppm})\end{array}$ & $\frac{207 \mathrm{~Pb}^{*}}{206 \mathrm{~Pb}^{*}}$ & $\pm \%$ & $\frac{207 \mathrm{~Pb}^{*}}{235 \mathrm{U}}$ & $\pm \%$ & $\frac{206 \mathrm{~Pb}^{*}}{238 \mathrm{U}}$ & $\pm \%$ & $\begin{array}{c}\text { err } \\
\text { corr }\end{array}$ & $\begin{array}{c}\frac{206 \mathrm{~Pb}}{238 \mathrm{U}} \\
\text { Age }\end{array}$ & \multicolumn{2}{|c|}{$\begin{array}{c}\frac{207 \mathrm{~Pb}}{206 \mathrm{~Pb}} \\
\text { Age }\end{array}$} & $\begin{array}{l}\text { Discordance } \\
(\%)\end{array}$ \\
\hline \multicolumn{16}{|c|}{ Garnet-sillimanite-biotite gneiss (QT1-12b) } \\
\hline $5.1 \mathrm{D}$ & 317 & 99 & 0.32 & 78 & 0.1222 & 1.00 & 4.811 & 2.6 & 0.2853 & 2.4 & 0.92 & $1618 \pm 34$ & 1989 & \pm 18 & 19 \\
\hline $6.1 \mathrm{RC}$ & 697 & 20 & 0.03 & 125 & 0.1148 & 0.80 & 3.300 & 2.3 & 0.2085 & 2.1 & 0.94 & $1221 \pm 24$ & 1876 & \pm 14 & 35 \\
\hline $7.1 \mathrm{R}$ & 425 & 65 & 0.16 & 96 & 0.1157 & 0.81 & 4.182 & 2.7 & 0.2624 & 2.5 & 0.95 & $1502 \pm 34$ & 1890 & \pm 15 & 21 \\
\hline 8.1D & 158 & 45 & 0.29 & 52 & 0.1243 & 1.20 & 6.491 & 2.5 & 0.3788 & 2.2 & 0.88 & $2070 \pm 39$ & 2019 & \pm 22 & -3 \\
\hline 9.1D & 233 & 45 & 0.20 & 68 & 0.1195 & 0.80 & 5.620 & 2.3 & 0.3412 & 2.2 & 0.94 & $1893 \pm 36$ & 1948 & \pm 14 & 3 \\
\hline 10.1D & 254 & 89 & 0.36 & 61 & 0.1229 & 0.92 & 4.702 & 2.4 & 0.2771 & 2.2 & 0.92 & $1577 \pm 31$ & 1999 & \pm 16 & 21 \\
\hline $10.2 \mathrm{RC}$ & 1263 & 542 & 0.44 & 85 & 0.1066 & 2.60 & 1.116 & 3.4 & 0.0759 & 2.2 & 0.63 & $471 \pm 10$ & 1743 & \pm 49 & 73 \\
\hline 11.1D & 293 & 449 & 1.58 & 116 & 0.1239 & 0.54 & 7.902 & 2.2 & 0.4624 & 2.2 & 0.97 & $2450 \pm 44$ & 2013 & \pm 10 & -22 \\
\hline $11.2 \mathrm{R}$ & 442 & 30 & 0.07 & 99 & 0.1137 & 1.40 & 4.071 & 2.6 & 0.2594 & 2.2 & 0.84 & $1487 \pm 29$ & 1859 & \pm 25 & 20 \\
\hline $12.1 \mathrm{D}$ & 236 & 66 & 0.29 & 56 & 0.1224 & 0.95 & 4.640 & 2.4 & 0.2750 & 2.2 & 0.92 & $1566 \pm 30$ & $1992=$ & \pm 17 & 21 \\
\hline $12.2 \mathrm{R}$ & 574 & 5 & 0.01 & 116 & 0.1117 & 0.60 & 3.604 & 2.3 & 0.2341 & 2.2 & 0.96 & $1356 \pm 27$ & 1827 & \pm 11 & 26 \\
\hline 13.1D & 118 & 31 & 0.27 & 35 & 0.1229 & 1.30 & 5.870 & 2.6 & 0.3468 & 2.3 & 0.87 & $1919 \pm 38$ & 1998 & \pm 23 & 4 \\
\hline \multicolumn{16}{|c|}{ Graphite-bearing two-mica two-feldspar gneiss (LT1-11) } \\
\hline $1.1 \mathrm{D}$ & 197 & 95 & 0.5 & 61 & 0.1240 & 0.76 & 6.181 & 2.5 & 0.3614 & 2.3 & 0.95 & $1989 \pm 40$ & 2015 & \pm 13 & 1 \\
\hline $1.2 \mathrm{R}$ & 561 & 15 & 0.03 & 87 & 0.1102 & 0.81 & 2.725 & 2.3 & 0.1793 & 2.1 & 0.93 & $1063 \pm 21$ & 1803 & \pm 15 & 41 \\
\hline 2.1D & 154 & 79 & 0.53 & 44 & 0.1245 & 0.93 & 5.672 & 2.4 & 0.3301 & 2.2 & 0.92 & $1839 \pm 35$ & 2022 & \pm 16 & 9 \\
\hline $2.2 \mathrm{R}$ & 802 & 9 & 0.01 & 122 & 0.1072 & 0.63 & 2.618 & 2.2 & 0.1771 & 2.1 & 0.96 & $1051 \pm 21$ & 1753 & \pm 12 & 40 \\
\hline 3.1D & 185 & 37 & 0.21 & 48 & 0.1232 & 0.79 & 5.161 & 2.3 & 0.3037 & 2.2 & 0.94 & $1710 \pm 33$ & 2004 & \pm 14 & 15 \\
\hline $4.1 \mathrm{R}$ & 1240 & 18 & 0.02 & 105 & 0.0847 & 0.79 & 1.147 & 2.3 & 0.0983 & 2.2 & 0.94 & $604 \pm 13$ & 1308 & \pm 15 & 54 \\
\hline $5.1 \mathrm{R}$ & 609 & 37 & 0.06 & 98 & 0.1106 & 1.00 & 2.842 & 2.4 & 0.1863 & 2.1 & 0.9 & $1102 \pm 21$ & 1809 & \pm 19 & 39 \\
\hline $5.2 \mathrm{D}$ & 342 & 205 & 0.62 & 84 & 0.1286 & 0.65 & 5.063 & 2.2 & 0.2853 & 2.1 & 0.96 & $1618 \pm 31$ & 2078 & \pm 11 & 22 \\
\hline $6.1 \mathrm{R}$ & 591 & 17 & 0.03 & 93 & 0.1127 & 0.75 & 2.823 & 2.3 & 0.1817 & 2.1 & 0.94 & $1076 \pm 21$ & 1844 & \pm 14 & 42 \\
\hline $6.2 \mathrm{D}$ & 225 & 57 & 0.26 & 60 & 0.1261 & 0.86 & 5.360 & 2.6 & 0.3084 & 2.4 & 0.94 & $1733 \pm 37$ & 2045 & \pm 15 & 15 \\
\hline $7.1 \mathrm{R}$ & 1075 & 44 & 0.04 & 111 & 0.0983 & 2.20 & 1.592 & 3 & 0.1174 & 2.1 & 0.71 & $716 \pm 15$ & 1592 & \pm 40 & 55 \\
\hline 7.2RC & 522 & 52 & 0.10 & 98 & 0.1176 & 0.91 & 3.524 & 2.3 & 0.2173 & 2.2 & 0.92 & $1267 \pm 25$ & 1921 & \pm 16 & 34 \\
\hline $8.1 \mathrm{R}$ & 981 & 34 & 0.04 & 100 & 0.0974 & 1.10 & 1.584 & 2.4 & 0.1179 & 2.1 & 0.89 & $719 \pm 15$ & 1575 & \pm 21 & 54 \\
\hline 9.1D & 332 & 66 & 0.21 & 84 & 0.1244 & 0.68 & 5.012 & 2.3 & 0.2923 & 2.2 & 0.95 & $1653 \pm 32$ & 2020 & \pm 12 & 18 \\
\hline $10.1 \mathrm{R}$ & 1286 & 35 & 0.03 & 99 & 0.0852 & 0.87 & 1.051 & 2.3 & 0.0895 & 2.2 & 0.93 & $553 \pm 12$ & 1319 & \pm 17 & 58 \\
\hline $10.2 \mathrm{D}$ & 1045 & 70 & 0.07 & 128 & 0.1019 & 0.62 & 2.005 & 2.2 & 0.1428 & 2.1 & 0.96 & $860 \pm 17$ & 1658 & \pm 12 & 48 \\
\hline 11.1D & 1023 & 15 & 0.02 & 79 & 0.1074 & 1.00 & 1.331 & 2.6 & 0.0898 & 2.4 & 0.92 & $554 \pm 13$ & 1756 & \pm 19 & 68 \\
\hline $11.2 \mathrm{R}$ & 782 & 6 & 0.01 & 83 & 0.1049 & 0.87 & 1.785 & 2.3 & 0.1234 & 2.1 & 0.93 & $750 \pm 15$ & 1712 & \pm 16 & 56 \\
\hline $12.1 \mathrm{R}$ & 1485 & 84 & 0.06 & 102 & 0.0810 & 0.95 & 0.889 & 2.3 & 0.0796 & 2.1 & 0.91 & $494 \pm 10$ & 1221 & \pm 19 & 60 \\
\hline 13.1D & 486 & 67 & 0.14 & 62 & 0.1150 & 1.10 & 2.331 & 2.4 & 0.1470 & 2.1 & 0.88 & $884 \pm 18$ & 1880 & \pm 21 & 53 \\
\hline $13.2 \mathrm{R}$ & 673 & 15 & 0.02 & 97 & 0.1039 & 0.79 & 2.401 & 2.3 & 0.1676 & 2.1 & 0.94 & $999 \pm 20$ & 1695 & \pm 15 & 41 \\
\hline $14.1 \mathrm{R}$ & 507 & 18 & 0.04 & 94 & 0.1089 & 0.88 & 3.226 & 2.4 & 0.2148 & 2.2 & 0.93 & $1254 \pm 25$ & 1782 & \pm 16 & 30 \\
\hline 15.1D & 219 & 55 & 0.26 & 57 & 0.1213 & 0.92 & 5.032 & 2.4 & 0.3010 & 2.2 & 0.92 & $1696 \pm 33$ & 1975 & \pm 16 & 14 \\
\hline 16.1D & 561 & 158 & 0.29 & 74 & 0.1236 & 0.81 & 2.616 & 2.3 & 0.1536 & 2.1 & 0.94 & $921 \pm 18$ & 2008 & \pm 14 & 54 \\
\hline & 950 & 8 & 0.01 & 95 & & 0.81 & 1.581 & 2.5 & 0.1156 & 2.4 & & $705 \pm 16$ & 1608 & \pm 15 & 56 \\
\hline $17.2 \mathrm{RC}$ & 416 & 57 & 0.14 & 81 & 0.1208 & 0.95 & 3.760 & 2.4 & 0.2258 & 2.2 & 0.91 & $1312 \pm 26$ & 1968 & \pm 17 & 33 \\
\hline \multicolumn{16}{|c|}{ Altered mica quartz-feldspar gneiss (QS1-18) } \\
\hline $1.1 \mathrm{C}$ & 195 & 58 & 0.31 & 52 & & & & 3.5 & & 3.4 & & \pm 52 & $61=$ & \pm 18 & 9 \\
\hline $1.2 \mathrm{C}$ & 1255 & 823 & 0.68 & 108 & 0.1103 & 0.61 & 1.521 & 2.4 & 0.1000 & 2.3 & 0.97 & $615 \pm 14$ & 1804 & \pm 11 & 66 \\
\hline $2.1 \mathrm{C}$ & 287 & 457 & 1.65 & 51 & & 0.78 & 3.397 & 2.5 & 0.2057 & 2.4 & 0.95 & $1206 \pm 27$ & 1953 & \pm 14 & 38 \\
\hline $2.2 \mathrm{C}+\mathrm{RC}$ & 794 & 376 & 0.49 & 92 & 0.1037 & 1.30 & 1.906 & 2.4 & 0.1332 & 2.1 & 0.86 & $806 \pm 16$ & 1692 & \pm 23 & 52 \\
\hline $3.1 \mathrm{RC}$ & 861 & 378 & 0.45 & 75 & & 2.50 & 1.207 & 3.3 & 0.0987 & 2.2 & 0.66 & $607 \pm 13$ & 1397 & \pm 48 & 57 \\
\hline $3.2 \mathrm{C}$ & 475 & 440 & 0.96 & 64 & 0.1113 & 1.00 & 2.400 & 2.3 & 0.1563 & 2.1 & 0.9 & $936 \pm 18$ & 1821 & \pm 19 & 49 \\
\hline $4.1 \mathrm{C}$ & 174 & 114 & 0.68 & 49 & & 0.8 & 5.452 & 2.3 & 0.3297 & 2.1 & 0.94 & $1837 \pm 34$ & 1955 & \pm 14 & 6 \\
\hline $5.1 \mathrm{C}$ & 370 & 178 & 0.50 & 82 & 0.1169 & 0.77 & 4.161 & 2.6 & 0.2582 & 2.4 & 0.95 & $1481 \pm 32$ & 1910 & \pm 14 & 22 \\
\hline $5.2 \mathrm{C}$ & 283 & 258 & 0.94 & 68 & & 0.65 & 4.536 & 2.2 & 0.2780 & 2.1 & 0.96 & $1581 \pm 29$ & 1931 & \pm 12 & 18 \\
\hline $6.1 \mathrm{C}$ & 214 & 126 & 0.61 & 53 & 0.1149 & 0.79 & 4.591 & 2.3 & 0.2900 & 2.1 & 0.94 & $1642 \pm 31$ & 1878 & \pm 14 & 13 \\
\hline 7.1RC & 1756 & 2599 & 1.53 & 100 & 0.0859 & 2.10 & 0.764 & 2.9 & 0.0645 & 2.1 & 0.7 & $403 \pm 8$ & 1335 & \pm 40 & 70 \\
\hline $7.2 \mathrm{C}$ & 697 & 381 & 0.56 & 86 & 0.1027 & 1.40 & 2.005 & 2.5 & 0.1415 & 2.1 & 0.82 & $853 \pm 17$ & 1674 & \pm 26 & 49 \\
\hline $8.1 \mathrm{R}$ & 337 & 77 & 0.24 & 74 & 0.1168 & 0.80 & 4.093 & 2.3 & 0.2542 & 2.1 & 0.94 & $1460 \pm 28$ & 1907 & \pm 14 & 23 \\
\hline $8.2 \mathrm{C}$ & 125 & 52 & 0.43 & 37 & 0.1240 & 1.00 & 5.973 & 2.4 & 0.3491 & 2.2 & 0.91 & $1930 \pm 37$ & 2015 & \pm 18 & 4 \\
\hline $9.1 \mathrm{C}$ & 147 & 36 & 0.25 & 46 & 0.1208 & 0.95 & 6.024 & 2.4 & 0.3613 & 2.2 & 0.92 & $1988 \pm 37$ & 1968 & \pm 17 & -1 \\
\hline $10.1 \mathrm{C}$ & 321 & 314 & 1.01 & 60 & 0.1130 & 0.99 & 3.358 & 2.3 & 0.2155 & 2.1 & 0.91 & $1258 \pm 24$ & 1848 & \pm 18 & 32 \\
\hline $11.1 \mathrm{C}$ & 179 & 152 & 0.88 & 43 & 0.1157 & 0.88 & 4.480 & 2.6 & 0.2808 & 2.4 & 0.94 & $1595 \pm 34$ & 1890 & \pm 16 & 16 \\
\hline
\end{tabular}


TABLE 2

(continued)

\begin{tabular}{|c|c|c|c|c|c|c|c|c|c|c|c|c|c|c|}
\hline Spot & $\begin{array}{c}\mathrm{U} \\
(\mathrm{ppm})\end{array}$ & $\begin{array}{c}\text { Th } \\
(\mathrm{ppm})\end{array}$ & $\frac{\mathrm{Th}}{\mathrm{U}}$ & $\begin{array}{l}206 \mathrm{~Pb}^{*} \\
\text { (ppm) }\end{array}$ & $\frac{207 \mathrm{~Pb}^{*}}{{ }^{206} \mathrm{~Pb}^{*}}$ & $\pm \%$ & $\frac{207 \mathrm{~Pb}^{*}}{235 \mathrm{U}}$ & $\pm \%$ & $\frac{206 \mathrm{~Pb}^{*}}{238 \mathrm{U}}$ & $\pm \%$ & $\begin{array}{l}\text { err } \\
\text { corr }\end{array}$ & $\begin{array}{c}\frac{206 \mathrm{~Pb}}{238 \mathrm{U}} \\
\text { Age }\end{array}$ & $\begin{array}{c}\frac{207 \mathrm{~Pb}}{206 \mathrm{~Pb}} \\
\text { Age }\end{array}$ & $\begin{array}{l}\text { Discordance } \\
(\%)\end{array}$ \\
\hline \multicolumn{15}{|c|}{ Altered mica quartz-feldspar gneiss (QS1-18) } \\
\hline $12.1 \mathrm{C}$ & 269 & 174 & 0.67 & 55 & 0.1135 & 10 & 3.679 & 2.4 & 0.2350 & 2.1 & 0.91 & $1361 \pm 26$ & $1856 \pm 18$ & 27 \\
\hline $12.2 \mathrm{R}$ & 831 & 198 & 0.25 & 83 & 0.0910 & 2.4 & 1.423 & 3.4 & 0.1134 & 2.4 & 0.7 & $692 \pm 16$ & $1447 \pm 47$ & 52 \\
\hline $13.1 \mathrm{C}$ & 210 & 63 & 0.31 & 62 & 0.1226 & 2.00 & 5.851 & 3.1 & 0.3461 & 2.4 & 0.77 & $1916 \pm 39$ & $1995 \pm 36$ & 4 \\
\hline 14.1RC & 741 & 190 & 0.27 & 85 & 0.0994 & 1.40 & 1.804 & 2.6 & 0.1317 & 2.2 & 0.85 & $797 \pm 16$ & $1612 \pm 26$ & 51 \\
\hline $15.1 \mathrm{R}$ & 984 & 342 & 0.36 & 108 & 0.0943 & 1.50 & 1.627 & 2.6 & 0.1252 & 2.1 & 0.81 & $760 \pm 15$ & $1514 \pm 29$ & 50 \\
\hline $16.1 \mathrm{R}$ & 1066 & 34 & 0.03 & 233 & 0.1131 & 0.70 & 3.931 & 2.2 & 0.2520 & 2.1 & 0.95 & $1449 \pm 27$ & $1850 \pm 13$ & 22 \\
\hline $17.1 \mathrm{R}$ & 471 & 174 & 0.38 & 90 & 0.1154 & 0.91 & 3.524 & 2.3 & 0.2215 & 2.1 & 0.92 & $1290 \pm 25$ & $1886 \pm 16$ & 32 \\
\hline $18.1 \mathrm{C}$ & 206 & 192 & 0.96 & 50 & 0.1179 & 0.81 & 4.612 & 3.2 & 0.2836 & 3.1 & 0.97 & $1609 \pm 45$ & $1924 \pm 14$ & 16 \\
\hline 19.1R & 725 & 61 & 0.09 & 80 & 0.0995 & 1.60 & 1.728 & 3.0 & 0.1260 & 2.6 & 0.85 & $765 \pm 18$ & $1614 \pm 30$ & 53 \\
\hline
\end{tabular}

Note: 1) Common lead corrected using measured ${ }^{204} \mathrm{~Pb}$; 2) ${ }^{206} \mathrm{~Pb} *$ is radiogenic lead; 3) Discordance $(\%)$ is defined as $\left[\left(1-\left({ }^{206} \mathrm{~Pb} /{ }^{238} \mathrm{U}\right)\right.\right.$ age $) /\left({ }^{267} \mathrm{~Pb} /{ }^{206} \mathrm{~Pb}\right)$ age $\left.] \times 100 ; 4\right)$ age in Ma; 5$) \mathrm{D}$ in samples QT1-12b and LT1-11 means detrital core of zircon, C in samples S2-5, H3-7 and QS1-18 means central domain of zircon, with no genetic implication, $\mathrm{RC}$ and $\mathrm{R}$ in all samples mean recrystallized zircon domain and zircon rim, respectively.

spots on the cores have $\mathrm{U}$ contents and $\mathrm{Th} / \mathrm{U}$ ratios ranging from 227 to $3637 \mathrm{ppm}$ (mostly $<1000 \mathrm{ppm}$ ) and 0.28 to 0.89 , respectively. They commonly show strong lead loss with only one analysis (14.1C) positioned close to concordia (fig. 5A) and having a ${ }^{207} \mathrm{~Pb} /{ }^{206} \mathrm{~Pb}$ age of $2404 \pm 4 \mathrm{Ma}$. The oldest core has an age of $2544 \pm 15 \mathrm{Ma}(22.1 \mathrm{C})$, but with a discordance of 30 percent. Based on the CL images and the data distribution pattern in the concordia diagram, we interpret the cores to reflect a Neoarchean to earliest Paleoproterozoic magmatic event. Two analyses on recrystallized cores record a restricted range of ${ }^{207} \mathrm{~Pb} /{ }^{206} \mathrm{~Pb}$ ages from $2426 \pm 13$ to $2450 \pm 12 \mathrm{Ma}$. Dark zircon rims exhibit a homogenous structure or have weak zoning in CL images (figs. 4A, 4B, and 4C), and eight analyses yielded higher $\mathrm{U}$ contents (1060-3918 ppm) and lower $\mathrm{Th} / \mathrm{U}$ ratios $(0.03-0.16)$ than the cores. These analyses are similarly discordant and spot 25.1R, which is the closest to concordia, has a ${ }^{207} \mathrm{~Pb} /{ }^{206} \mathrm{~Pb}$ age of $2278 \pm 13 \mathrm{Ma}$. Several zircons (grain 7 in fig. 4B; grain 12 in fig. 4C) have light gray outer rims, but these were too narrow to analyze.

\section{Banded Two-Mica K-Feldspar Gneiss (H3-7)}

The zircons are similar in shape to those of sample S2-5 and also commonly show core-rim structures (figs. 4D, 4E, 4F, and 4G). The cores exhibit oscillatory or banded zoning, with some having a fairly homogenous structure, possibly the result of recrystallization. The dark rim domains are either homogenous or show weak oscillatory zoning in CL images (fig. 4G). Twenty one analyses were carried out on 15 zircons (table 2). Eleven analyses on the cores have $\mathrm{U}$ contents and $\mathrm{Th} / \mathrm{U}$ ratios of 47 to 492 ppm and 0.22 to 1.57 , respectively. Most show lead loss to variable degrees, with three spots $(4.1 \mathrm{C}, 5.1 \mathrm{C}, 15.1 \mathrm{C})$ positioned close to concordia and having ${ }^{207} \mathrm{~Pb} /{ }^{206} \mathrm{~Pb}$ ages of $\sim 2.0 \mathrm{Ga}$ (fig. 5B). Four analyses on recrystallized cores exhibit a similar distribution in the concordia diagram as the non-recrystallized core analyses. The rims have much higher $\mathrm{U}$ contents (705-2511 ppm) and lower $\mathrm{Th} / \mathrm{U}$ ratios (0.02-0.34) (6 analyses) than the cores and commonly experienced stronger lead loss. However, they are aligned along a discordia line with an upper intercept age of $1882 \pm 45 \mathrm{Ma}$ (MSWD = 1.9). Although rather imprecise, this age is interpreted to reflect a metamorphic and/or anatetic event, as is evident from the hand sample (fig. 3B). 

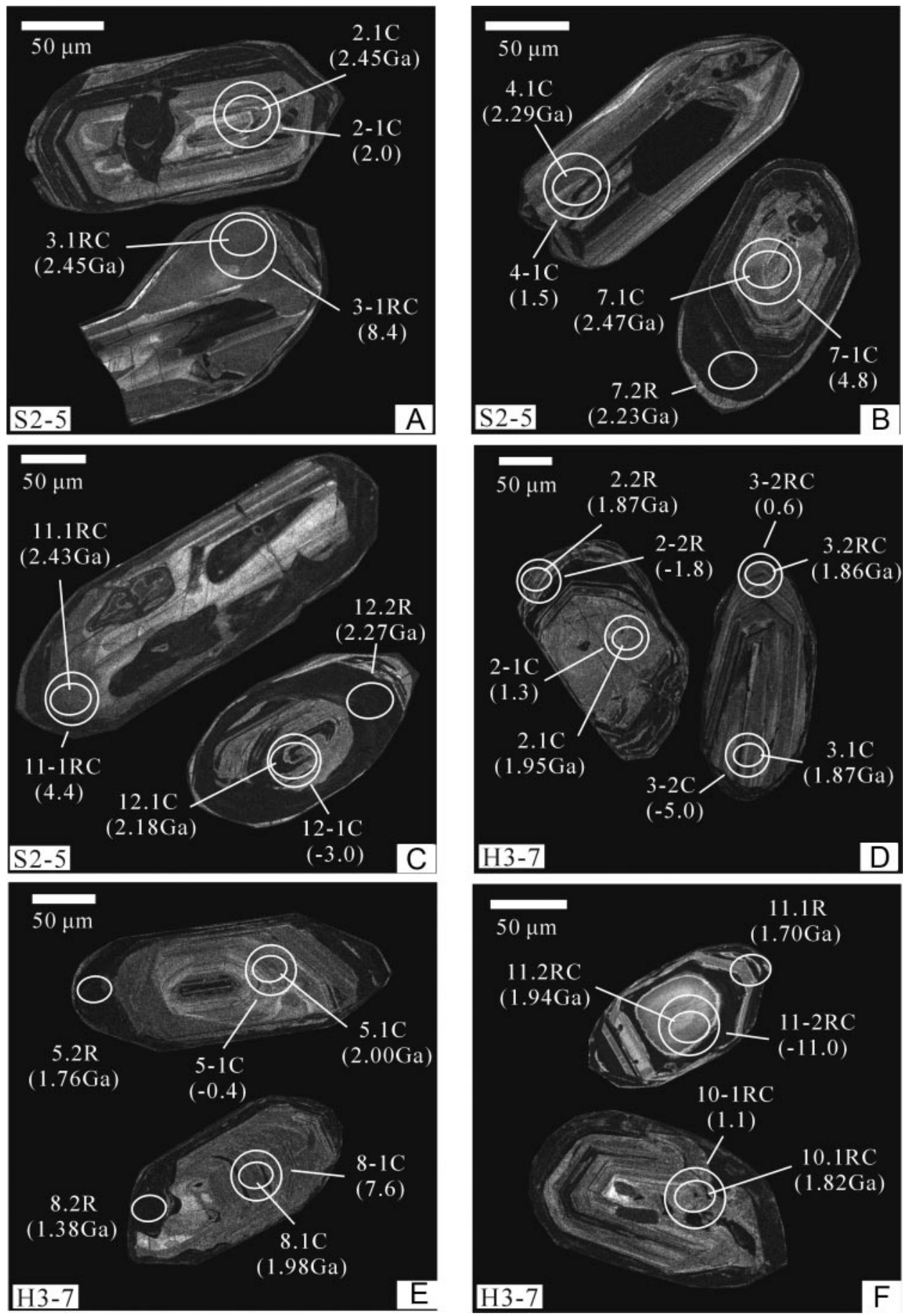

Fig. 4. Cathodoluminescence images of zircons from Paleoproterozoic rocks from drill cores beneath the Ordos Basin. (A)-(C) Biotite K-feldspar gneiss (S2-5); (D)-(G) banded two-mica K-feldspar gneiss (H3-7); (H)-(J) garnet-sillimanite-biotite gneiss (QT1-12b); (K)-(N) graphite-bearing two-mica two-feldspar gneiss (LT1-11); (O)-(R) altered mica quartz-feldspar gneiss (QS1-18). D in samples QT1-12b and LT1-11 means detrital core of zircon, C in samples S2-5, H3-7 and QS1-18 means central domain of zircon, with no genetic implication, $\mathrm{RC}$ and $\mathrm{R}$ in all samples mean recrystallized zircon domain and zircon rim, respectively. 

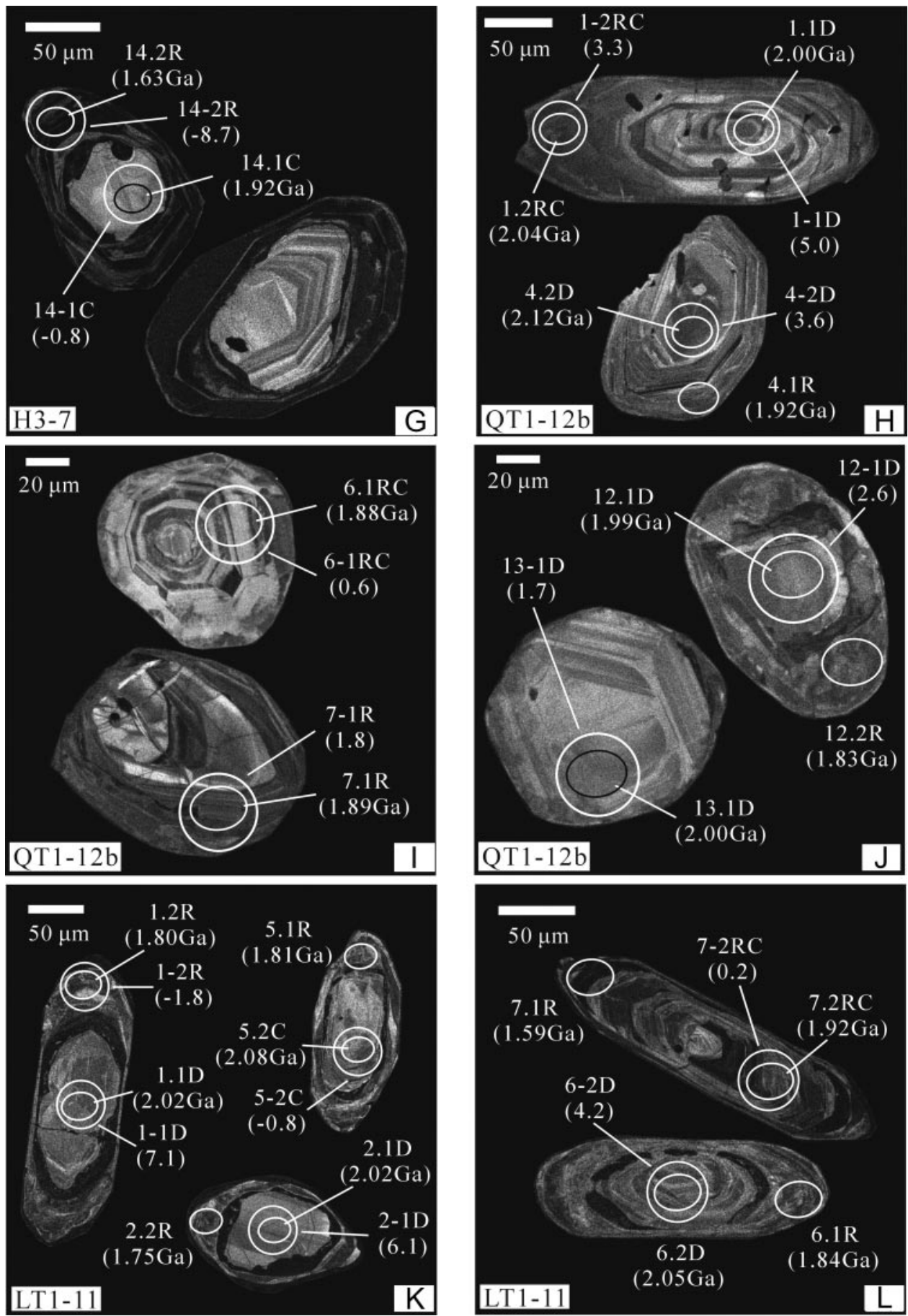

Fig. 4 (continued). 

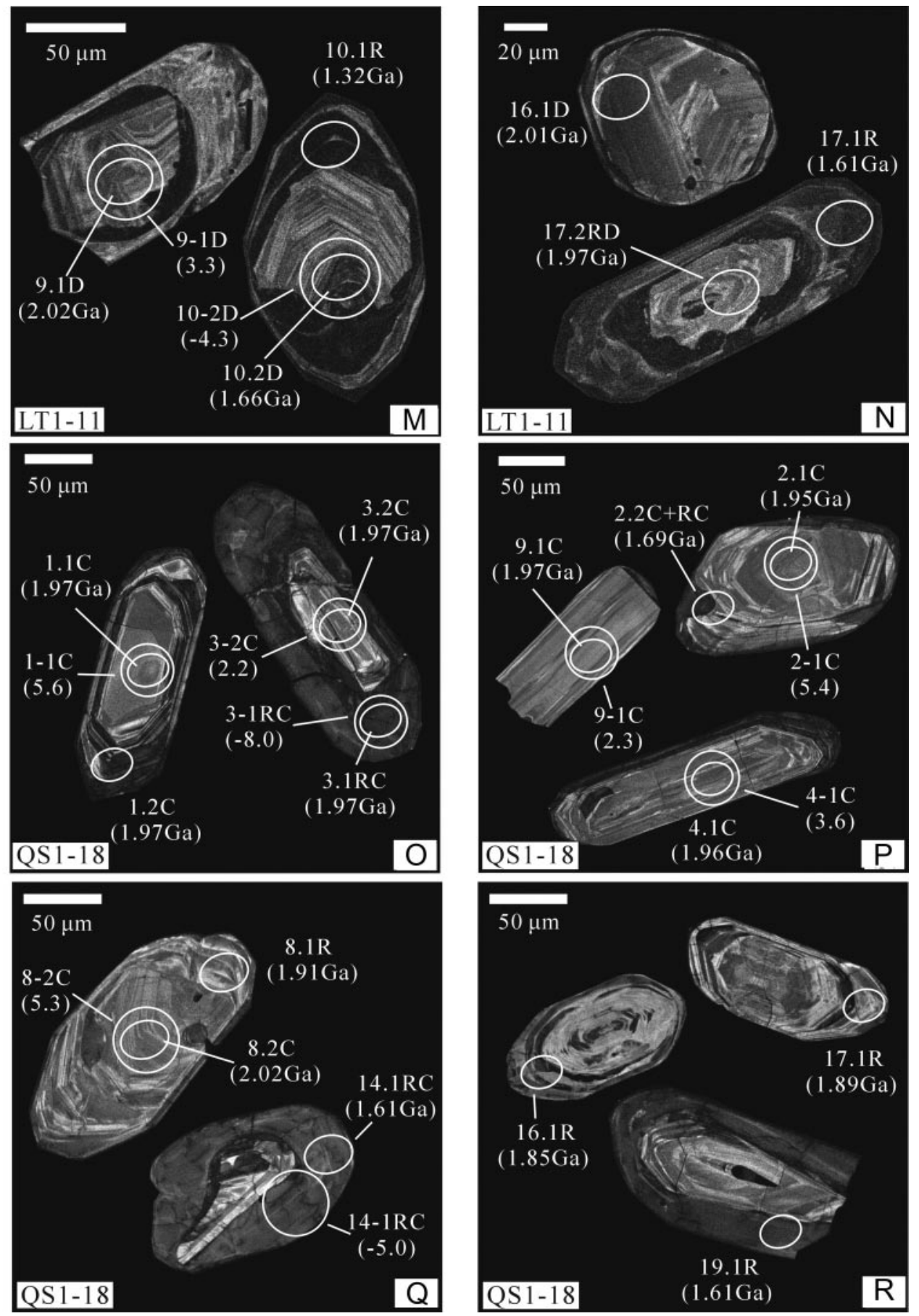

Fig. 4 (continued). 

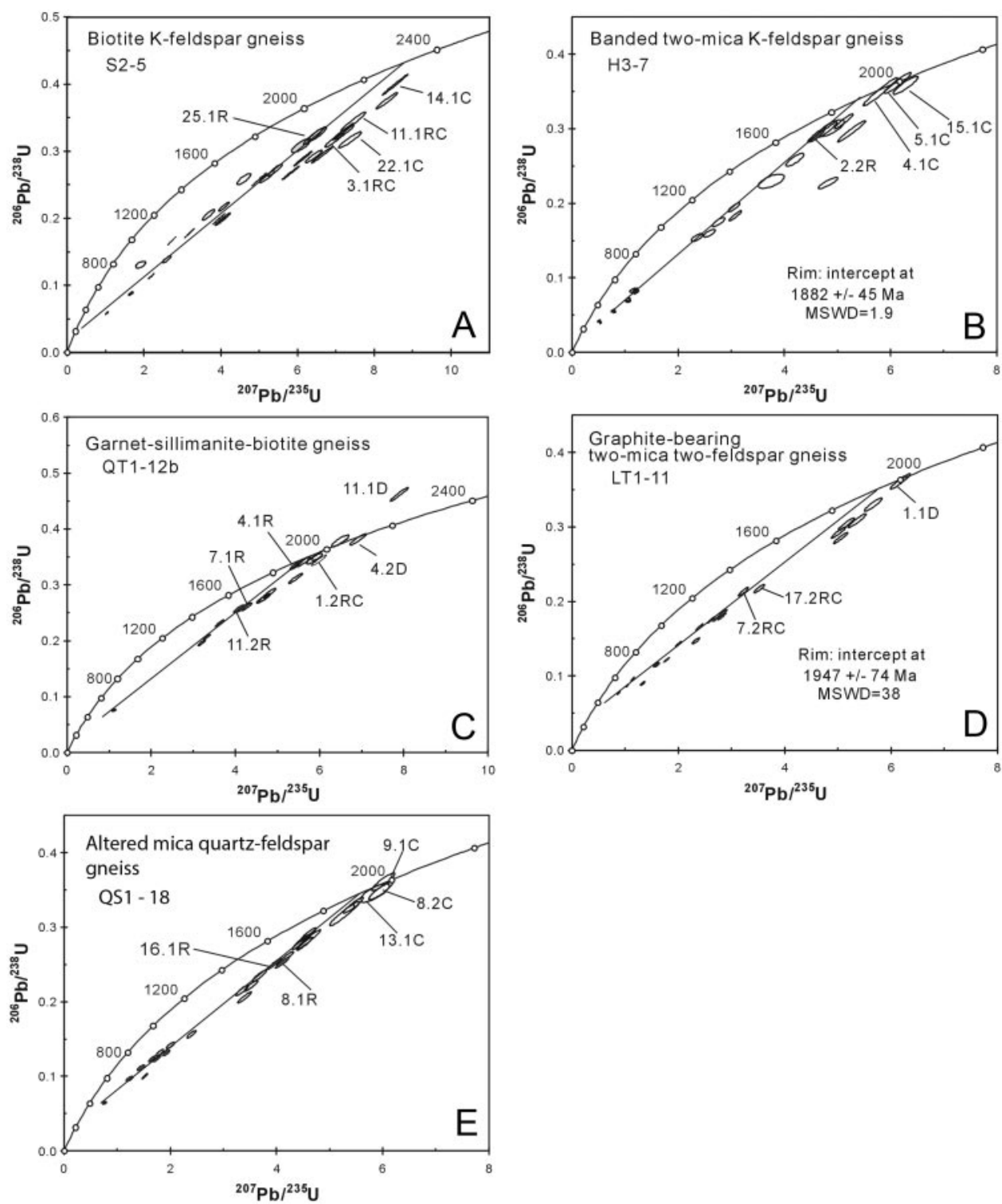

Fig. 5. Concordia diagrams showing SHRIMP zircon data for Paleoproterozoic rocks obtained from drill cores beneath the Ordos Basin. (A) Biotite K-feldspar gneiss (S2-5); (B) banded two-mica K-feldspar gneiss (H3-7); (C) garnet-sillimanite-biotite gneiss (QT1-12b); (D) graphite-bearing two-mica two-feldspar gneiss (LT1-11); (E) altered mica quartz-feldspar gneiss (QS1-18).

\section{Garnet-Sillimanite-Biotite Gneiss (QT1-12b)}

Most zircon grains are round or stubby, but some are elongate and some show apparent core-rim structures (figs. 4H, 4I, and 4J). The detrital cores exhibit magmatic zoning with some showing evidence of recrystallization. The rims tend to be more homogeneous or show weak zoning. A total of nineteen analyses were made on 13 grains (table 2). Eleven analyses on core domains yielded $\mathrm{U}$ contents and $\mathrm{Th} / \mathrm{U}$ ratios 
of 93 to $382 \mathrm{ppm}$ and 0.20 to 1.58 , respectively. Although most analyses show lead loss to variable degrees, some are located on or near concordia with ${ }^{207} \mathrm{~Pb} /{ }^{206} \mathrm{~Pb}$ ages ranging from $2003 \pm 24$ to $2118 \pm 14$ Ma. Recrystallized cores (4 analyses) show a similar age distribution to the "cores." Four rim analyses yielded U contents of 442 to $574 \mathrm{ppm}$ and $\mathrm{Th} / \mathrm{U}$ ratios of 0.01 to 0.27 . One analysis (4.1R) plots close to concordia and has a ${ }^{207} \mathrm{~Pb} /{ }^{206} \mathrm{~Pb}$ age of $1920 \pm 14 \mathrm{Ma}$ that is taken to record the age of the late Paleoproterozoic tectono-thermal event.

\section{Graphite-Bearing Two-Mica Two-Feldspar Gneiss (LT1-11)}

The zircons are long-prismatic or stubby to rounded in shape with rounded terminations, and some show core-rim structures in CL images (figs. $4 \mathrm{~K}, 4 \mathrm{~L}, 4 \mathrm{M}$, and $4 \mathrm{~N})$. Oscillatory zoning suggests that the cores of these detrital grains are of magmatic origin. The rims vary in width and are commonly darker in CL images and lack internal structure or else show weak zoning. Twenty six analyses were carried out on 17 grains (table 2), of which eleven core analyses have $\mathrm{U}$ contents and $\mathrm{Th} / \mathrm{U}$ ratios of 154 to $1045 \mathrm{ppm}$ and 0.07 to 0.62 , respectively. Most cores have Th/U ratios higher than 0.1 , although cores with $\mathrm{Th} / \mathrm{U}<0.1$ appear to have undergone recrystallization, with magmatic zoning still present locally (for example, grain 7 in fig. $4 \mathrm{~L}$ ). The cores show lead loss with only one analysis (1.1D) plotting on concordia, and this has a ${ }^{207} \mathrm{~Pb} /{ }^{206} \mathrm{~Pb}$ age of $2015 \pm 13 \mathrm{Ma}$. Two analyses $(7.2 \mathrm{RC}, 17.2 \mathrm{RC})$ on recrystallized domains recorded ${ }^{207} \mathrm{~Pb} /{ }^{206} \mathrm{~Pb}$ ages of $1921 \pm 17$ and $1968 \pm$ Ma, respectively. Thirteen rim analyses have $\mathrm{U}$ contents ranging from 507 to $1485 \mathrm{ppm}$ and $\mathrm{Th} / \mathrm{U}$ ratios of 0.01 to 0.06 . The rims commonly show stronger lead loss than the cores and the upper concordia intercept age of a poorly defined discordia line, including all rim data points, is $1947 \pm 74 \mathrm{Ma}(\mathrm{MSWD}=38)$.

\section{Altered Mica Quartz-Feldspar Gneiss (QS1-18)}

The zircons are similar in shape to those of sample LT1-11, and some also display core-rim structures (figs. 4O, 4P, 4Q, and $4 \mathrm{R}$ ). The cores show oscillatory or banded zoning, with some revealing partial recrystallization, and are cross-cut by rim domains (for example, grain 8 in fig. 4Q). A total of twenty-six analyses were made on 19 grains (table 2), of which 16 core analyses yielded $\mathrm{U}$ contents of 125 to $1255 \mathrm{ppm}$ and $\mathrm{Th} / \mathrm{U}$ ratios of 0.25 to 1.65 . As in the other samples, most analyses show strong lead loss, but some plot on or near concordia and define ${ }^{207} \mathrm{~Pb} /{ }^{206} \mathrm{~Pb}$ ages of $2015 \pm 18$ to $1968 \pm 17$ Ma (8.2C, 9.1C, 13.1C). Three recrystallized core domains show strong lead loss. Six analyses on rim domains reveal $\mathrm{U}$ contents of 337-1066 ppm and $\mathrm{Th} / \mathrm{U}$ ratios of 0.03 to 0.38. Similar to sample LT1-11, the age of the zircon rims suggests formation during a late Paleoproterozoic tectono-thermal event, although no precise metamorphic age could be assigned because of the strong lead loss.

\section{HF-IN-ZIRCON ISOTOPIC SIGNATURES}

Wherever possible, the Hf isotopes were obtained on sites previously analyzed on SHRIMP II for U-Pb. The results are given in table 3, are shown in figures $6 \mathrm{~A}-6 \mathrm{E}$, and are summarized in figure $6 \mathrm{~F}$. Cores (17 analyses) from the early Paleoproterozoic or late Neoarchean (see below) sample $(\mathrm{S} 2-5)$ have $\varepsilon_{\mathrm{Hf}}(\mathrm{t})$ values $\left(\mathrm{t}\right.$ is the ${ }^{207} \mathrm{~Pb} /{ }^{206} \mathrm{~Pb}$ age of the analytical spot) of -8.8 to 6.2 , and Hf crustal model ages of 2606 to $3221 \mathrm{Ma}$. Five analyses on recrystallized core and rim domains yielded $\varepsilon_{\mathrm{Hf}}(\mathrm{t})$ values of -4.9 to 8.4 and Hf crustal model ages of to 2447 to $2866 \mathrm{Ma}$ and are overall slightly younger than the magmatic core domains.

The cores (44 analyses in total) from the late or middle-late Paleoproterozoic (see below) samples (H3-7, QT1-12b, LT1-11, QS1-18) have similar Hf isotopic compositions with $\varepsilon_{\mathrm{Hf}}(\mathrm{t})$ values and $\mathrm{Hf}$ crustal model ages of -9.1 to +10.1 and 2039 to 3068 $\mathrm{Ma}$, respectively. The recrystallized cores and rim domains (17 analyses) have $\varepsilon_{\mathrm{Hf}}(\mathrm{t})$ 
TABLE 3

Hf isotope data for zircons from Paleoproterozoic metasedimentary rocks obtained from drill cores beneath the Ordos Basin

\begin{tabular}{|c|c|c|c|c|c|c|c|c|c|c|c|c|}
\hline $\begin{array}{l}\mathrm{Hf} \\
\text { spot }\end{array}$ & $\begin{array}{l}\text { Age } \\
\text { spot }\end{array}$ & $\begin{array}{l}\text { Age } \\
(\mathrm{Ma})\end{array}$ & ${ }^{{ }^{176} \mathrm{Yb}}$ & ${ }^{\frac{176}{{ }^{177} \mathrm{Lu}}} \mathrm{Hf}$ & ${ }^{\frac{176}{177} \mathrm{Hf}}$ & $2 \sigma$ & $\varepsilon H f(t)$ & $2 \sigma$ & $\begin{array}{r}t_{\mathrm{DM} 1} \\
(\mathrm{Ma}) \\
\end{array}$ & $2 \sigma$ & $\begin{array}{c}t_{\mathrm{DM} 2(\mathrm{CC})} \\
(\mathrm{Ma})\end{array}$ & $2 \sigma f_{\mathrm{Lu}}$ \\
\hline \multicolumn{13}{|c|}{ Biotite K-feldspar gneiss (S2-5) } \\
\hline $1-1 \mathrm{C}$ & $1.1 \mathrm{C}$ & 2087 & 0.078582 & 0.002132 & 0.28129 & 0.000026 & -8.8 & 0.9 & 2821 & 72 & 3221 & $110-0.94$ \\
\hline $2-1 \mathrm{C}$ & $2.1 \mathrm{C}$ & 2450 & 0.059465 & 0.00126 & 0.281335 & 0.000022 & 2 & 0.8 & 2695 & 60 & 2838 & $95-0.96$ \\
\hline $3-1 \mathrm{RC}$ & $3.1 \mathrm{RC}$ & 2450 & 0.093145 & 0.002532 & 0.281575 & 0.00003 & 8.4 & 1.1 & 2448 & 86 & 2447 & $131-0.92$ \\
\hline $4-1 \mathrm{C}$ & $4.1 \mathrm{C}$ & 2291 & 0.044449 & 0.000942 & 0.281405 & 0.00002 & 1.5 & 0.7 & 2578 & 56 & 2748 & $89-0.97$ \\
\hline $5-1 \mathrm{C}$ & $5.1 \mathrm{C}$ & 2193 & 0.070985 & 0.001635 & 0.281401 & 0.000023 & -1.9 & 0.8 & 2631 & 63 & 2878 & $99-0.95$ \\
\hline $7-1 \mathrm{C}$ & $7.1 \mathrm{C}$ & 2466 & 0.066108 & 0.001527 & 0.281417 & 0.000019 & 4.8 & 0.7 & 2601 & 54 & 2678 & $84-0.95$ \\
\hline $8-1 \mathrm{C}$ & $8.1 \mathrm{C}$ & 2269 & 0.048162 & 0.001266 & 0.281367 & 0.000029 & -0.9 & 1 & 2653 & 79 & 2875 & $124-0.96$ \\
\hline $9-1 \mathrm{C}$ & $9.1 \mathrm{C}$ & 2448 & 0.079564 & 0.002248 & 0.281440 & 0.000027 & 4 & 0.9 & 2620 & 75 & 2713 & $116-0.93$ \\
\hline $10-1 \mathrm{C}$ & $10.1 \mathrm{C}$ & 2280 & 0.070891 & 0.002083 & 0.281361 & 0.00003 & -2.1 & 1.1 & 2719 & 84 & 2959 & $130-0.94$ \\
\hline $11-1 \mathrm{RC}$ & $11.1 \mathrm{RC}$ & 2426 & 0.022215 & 0.000496 & 0.281383 & 0.000016 & 4.4 & 0.6 & 2578 & 44 & & $71-0.99$ \\
\hline $12-1 C$ & $12.1 \mathrm{C}$ & 2181 & 0.062170 & 0.001448 & 0.281371 & 0.000023 & -3 & 0.8 & 2660 & 63 & & $99-0.96$ \\
\hline $12-2 \mathrm{R}+$ & $12.2 \mathrm{R}+$ & 2268 & 0.049238 & 0.001363 & 0.281405 & 0.00 & 0.3 & 0.8 & 2607 & 64 & 02 & $100-0.96$ \\
\hline $13-1 \mathrm{C}$ & $13.1 \mathrm{C}$ & 2438 & 0.076631 & 768 & 0.281363 & 0.000 & 1.9 & 1.1 & 93 & 87 & & $136-0.95$ \\
\hline $14-1 \mathrm{C}$ & $14.1 \mathrm{C}$ & 2404 & 0.118337 & 0.002909 & 0.281328 & 0.00003 & -2 & 1.1 & 2828 & 86 & & $130-0.91$ \\
\hline $16-1 \mathrm{C}$ & $16.1 \mathrm{C}$ & 2493 & 0.086378 & 325 & 0.281432 & 0.0 & 4.6 & 1 & & 77 & & $119-0.93$ \\
\hline $17-1 \mathrm{R}$ & $17.1 \mathrm{R}$ & 2092 & 0.090387 & 551 & 0.281486 & 0.00 & -2.4 & 1 & & 84 & & $128-0.92$ \\
\hline $18-1 R$ & IR & 193 & 0.137662 & 833 & 0.281555 & 0.0 & -4.9 & 0.6 & & 53 & & $78-0.88$ \\
\hline $19-1 \mathrm{C}$ & IC & 2059 & 0.153032 & 206 & 0.2 & 0. & 0.9 & 0.7 & & 62 & & $\begin{array}{lll}90 & -0.87\end{array}$ \\
\hline $20-1 \mathrm{C}$ & $20.1 \mathrm{C}$ & 2433 & 0.054926 & 333 & 0.281314 & 0.0 & 0.8 & 0.7 & & 57 & & $90-0.96$ \\
\hline $22-1 C$ & $1 \mathrm{C}$ & 2514 & 0.074592 & & & & 6.2 & 0.7 & & 53 & & $83-0.95$ \\
\hline $24-1 \mathrm{C}$ & $1 \mathrm{C}$ & 2393 & 0.074318 & & & & 0.3 & 0.7 & & 52 & & $82-0.95$ \\
\hline $27-2 \mathrm{C}$ & $27.2 \mathrm{C}$ & 2099 & 0.073909 & 594 & 0.2 & & -1.6 & 0.9 & & 69 & 2790 & $108-0.95$ \\
\hline \multicolumn{13}{|c|}{ Banded two-mica K-feldspar gneiss (H3-7) } \\
\hline $1-1 \mathrm{C}$ & $1.1 \mathrm{C}$ & 1919 & 0.175601 & 11 & & & -3.3 & 0.7 & & 59 & & $88-0.91$ \\
\hline $1 \mathrm{RC}$ & रC & 2 & 270 & 83 & 36 & & 1.3 & 0.7 & & 56 & & $87-0.95$ \\
\hline $2 \mathrm{R}$ & $\mathrm{R}$ & 4 & 4851 & 637 & 1635 & 19 & -1.8 & 0.7 & 8 & 54 & 2626 & $82-0.92$ \\
\hline $1 \mathrm{C}$ & C & 72 & 0348 & 287 & 0.281727 & 0.0 & 0.6 & 0.9 & 6 & 76 & 2476 & $113-0.9$ \\
\hline $\mathrm{RC}$ & $\mathrm{RC}$ & 1 & 2157 & 918 & 667 & & -5 & 1.1 & & 98 & 2816 & $135-0.82$ \\
\hline $\mathrm{C}$ & & 63 & 4331 & 231 & 0.281358 & 0.0 & -7.9 & 0.7 & 26 & 56 & 3068 & $89-0.96$ \\
\hline $\mathrm{C}$ & & 01 & 3159 & 76 & 559 & 0.0 & -0.4 & 0.8 & 8 & 65 & 2638 & $102-0.95$ \\
\hline $\mathrm{C}$ & & 1 & 079 & 09 & 55 & 0.0 & 0 & 0.8 & & 62 & 3 & $95-0.93$ \\
\hline $\mathrm{C}$ & & 2 & 0.071063 & 84 & 789 & 0.0 & 7.6 & 0.7 & 20 & 59 & 2130 & $93-0.96$ \\
\hline $1 \mathrm{C}$ & & 5 & 0774 & 37 & 0.281703 & 0.0 & 1.1 & 0.8 & 7 & 61 & 2403 & $96-0.96$ \\
\hline $\mathrm{RC}$ & $\mathrm{RC}$ & 5 & 571 & 72 & 521 & 0.0 & -11 & 1.3 & 2 & 123 & 3235 & $161-0.77$ \\
\hline $1 \mathrm{C}$ & & 25 & 9804 & 77 & 0.281790 & 0.00 & 6 & 0.8 & 2091 & 60 & 2184 & $93-0.95$ \\
\hline lC & & 22 & 0.058489 & 194 & 582 & 0.0 & -0.8 & 0.9 & 2351 & 70 & 2602 & $111-0.96$ \\
\hline $\mathrm{R}$ & & 531 & 3752 & 05 & 908 & 0.0 & -8.7 & 1 & 2782 & 119 & 2866 & $128-0.6$ \\
\hline $1 \mathrm{C}$ & IC & 559 & 0.091399 & 27 & 0.281831 & 0.00 & 10.1 & 0.9 & 2046 & 68 & 2039 & $106-0.94$ \\
\hline $16-1 \mathrm{RC}$ & 16.1RC & 1909 & 0.216417 & 0.003757 & 0.281700 & 0.00 & -0.2 & 0.9 & 2347 & 71 & 2554 & $105-0.89$ \\
\hline \multicolumn{13}{|c|}{ Garnet-sillimanite-biotite gneiss (QT1-12b) } \\
\hline $1-1 \mathrm{D}$ & $1.1 \mathrm{D}$ & 2003 & 0.035015 & 04 & & & 5 & 0.7 & & 54 & & $86-0.98$ \\
\hline $1-2 \mathrm{RC}$ & $1.2 \mathrm{RC}$ & 038 & 0.029759 & 00917 & 0.281613 & 0.00 & 3.3 & 0.7 & 2292 & 55 & 2443 & $87-0.97$ \\
\hline $2-1 \mathrm{D}$ & & 2048 & 0.083546 & & 0.281751 & & 6.9 & 0.7 & 2165 & 57 & 2229 & $\begin{array}{ll}88 & -0.94\end{array}$ \\
\hline $3-2 \mathrm{D}$ & & 1940 & 0.053987 & & 0.281622 & 0.00 & 1.2 & 0.7 & 2291 & 53 & 2497 & $84-0.97$ \\
\hline $4-2 \mathrm{D}$ & $4.2 \mathrm{D}$ & 2118 & 0.037841 & 0.000866 & 0.281570 & 0.000 & 3.6 & 0.7 & 2348 & 51 & 2486 & $82-0.97$ \\
\hline $6-1 \mathrm{RC}$ & & 07 & 0.003059 & 0.000072 & 0.281608 & & 0.6 & 0.5 & 2249 & 39 & 2484 & $64-1$ \\
\hline $7-1 \mathrm{R}$ & & 0 & 0.020779 & & 0.281653 & & 1.8 & 0.6 & 2218 & 48 & 2418 & $\begin{array}{ll}77 & -0.98\end{array}$ \\
\hline $8-1 \mathrm{D}$ & & 2019 & 0.077380 & 0.002033 & 0.281608 & 0.000 & 1.1 & 0.8 & 2368 & 67 & 2559 & $104-0.94$ \\
\hline & & 1948 & 0.092365 & & 0.281732 & & 3.5 & 0.8 & & 61 & 2358 & $93-0.93$ \\
\hline $10-1 \mathrm{D}$ & & 1999 & & & 0.28 & 0.0 & 5.1 & 0.5 & 2190 & 42 & 2302 & $66-0.97$ \\
\hline $10-2 \mathrm{RC}$ & $10.2 \mathrm{RC}$ & 1743 & 0.057941 & 20 & 0.281671 & 0.000024 & -2.3 & 0.8 & 2266 & 67 & 2557 & $105-0.95$ \\
\hline & & 2013 & 0.069272 & & 0.281647 & 0.00 & 2.5 & 0.7 & 2307 & 57 & 2470 & $88-0.94$ \\
\hline & & 1992 & 0.109771 & & 0.281701 & & 2.6 & 0.6 & 2292 & 52 & 2445 & $78-0.91$ \\
\hline $13-1 \mathrm{D}$ & 13.1D & 1998 & 0.054927 & 0.001506 & 0.281615 & 0.000029 & 1.7 & 1 & 2325 & 80 & 2511 & $126-0.95$ \\
\hline
\end{tabular}


TABLE 3

(continued)

\begin{tabular}{|c|c|c|c|c|c|c|c|c|c|c|c|c|}
\hline $\begin{array}{l}\mathrm{Hf} \\
\text { spot }\end{array}$ & $\begin{array}{l}\text { Age } \\
\text { spot }\end{array}$ & $\begin{array}{l}\text { Age } \\
(\mathrm{Ma}) \\
\end{array}$ & $\frac{{ }^{176} \mathrm{Yb}}{{ }^{177} \mathrm{Hf}}$ & $\frac{{ }^{176} \mathrm{Lu}}{{ }^{177} \mathrm{Hf}}$ & $\frac{{ }^{176} \mathrm{Hf}}{{ }^{177} \mathrm{Hf}}$ & $2 \sigma$ & $\varepsilon \mathrm{Hf}(\mathrm{t}) 2 \sigma$ & $\begin{array}{r}t_{\mathrm{DM} 1} \\
(\mathrm{Ma}) \\
\end{array}$ & $2 \sigma$ & $\begin{array}{c}\mathrm{t}_{\mathrm{DM} 2(\mathrm{CC})} \\
(\mathrm{Ma}) \\
\end{array}$ & $2 \sigma$ & $f_{\mathrm{Lu}}$ \\
\hline \multicolumn{13}{|c|}{ Graphite-bearing two-mica two-feldspar gneiss (LT1-11) } \\
\hline $1-1 \mathrm{D}$ & $1.1 \mathrm{D}$ & 2015 & 0.096570 & 0.002000 & 0.281776 & 0.000020 & 7.10 .7 & 2128 & 58 & 2190 & 89 & -0.94 \\
\hline $1-2 \mathrm{R}$ & $1.2 \mathrm{R}$ & 1803 & 0.106452 & 0.003232 & 0.281696 & 0.000023 & -1.80 .8 & 2319 & 66 & 2575 & 99 & -0.9 \\
\hline $2-1 \mathrm{D}$ & $2.1 \mathrm{D}$ & 2022 & 0.091032 & 0.001931 & 0.281741 & 0.000021 & 6.10 .8 & 2174 & 61 & 2258 & 94 & -0.94 \\
\hline $5-2 \mathrm{D}$ & $5.2 \mathrm{D}$ & 2078 & 0.082526 & 0.001925 & 0.281513 & 0.000020 & -0.80 .7 & 2494 & 55 & 2724 & 85 & -0.94 \\
\hline $6-2 \mathrm{D}$ & $6.2 \mathrm{D}$ & 2045 & 0.080597 & 0.002106 & 0.281681 & 0.000021 & 4.20 .8 & 2269 & 60 & 2392 & 93 & -0.94 \\
\hline $7-2 \mathrm{RC}$ & $7.2 \mathrm{RC}$ & 1921 & 0.106307 & 0.002937 & 0.281673 & 0.000018 & 0.20 .6 & 33 & 51 & 543 & 77 & -0.91 \\
\hline $9-1 \mathrm{D}$ & 9.1D & 2020 & 0.055812 & 0.001386 & 0.281642 & 0.000017 & 3.30 .6 & 2280 & 49 & 429 & 76 & -0.96 \\
\hline $10-2 \mathrm{D}$ & $10.2 \mathrm{D}$ & 1658 & 0.070114 & 0.001593 & 0.281659 & 0.000017 & -4.30 .6 & 2269 & 47 & 2616 & 74 & -0.95 \\
\hline 11-1D & $1 \mathrm{D}$ & 1756 & 0.073952 & 0.001978 & 0.281467 & 0.000030 & -9.41 .1 & 2562 & 84 & 3006 & 130 & 0.94 \\
\hline 13-1D & 13.1D & 1880 & 0.090331 & 0.002518 & 0.281689 & 0.000030 & 0.41 .1 & 284 & 85 & 2497 & 130 & 0.92 \\
\hline \multicolumn{13}{|c|}{ Altered mica quartz-feldspar gneiss (QS1-18) } \\
\hline $1-1 C$ & $1.1 \mathrm{C}$ & 1961 & 0.077196 & 0.001556 & 0.281750 & & 5.60 .7 & 2140 & 55 & 2242 & 86 & -0.95 \\
\hline $2-1 C$ & $2.1 \mathrm{C}$ & 1953 & 0.065404 & 0.001304 & 0.281741 & 0.000021 & 5.40 .7 & 2139 & 58 & 2246 & 91 & -0.96 \\
\hline $3-1 \mathrm{RC}$ & $3.1 \mathrm{RC}$ & 1397 & 0.040080 & 0.000684 & 0.281688 & 0.000017 & -8 & 2177 & 46 & 2648 & 74 & -0.98 \\
\hline $3-2 \mathrm{C}$ & $3.2 \mathrm{C}$ & 1821 & 0.076697 & 0.001370 & 0.281734 & 0.000017 & 2.20 .6 & 2151 & 47 & 2342 & 73 & -0.96 \\
\hline $4-1 C$ & $4.1 \mathrm{C}$ & 1955 & 0.085714 & 0.001736 & 0.281704 & 0.000018 & 3.60 .6 & 2215 & 51 & 2360 & 80 & -0.95 \\
\hline $5-2 \mathrm{C}$ & $5.2 \mathrm{C}$ & 1931 & 0.072102 & 0.001422 & 0.281714 & 0.000018 & 3.80 .6 & 2183 & 49 & 2327 & 77 & -0.96 \\
\hline $6-1 \mathrm{C}$ & $6.1 \mathrm{C}$ & 1878 & 0.064059 & 0.001340 & 0.281713 & 0.000019 & 2.70 .7 & 2179 & 54 & 2353 & 85 & -0.96 \\
\hline 7-1RC & 7.1RC & 1335 & 0.026282 & 0.000542 & 0.281640 & 0.000016 & -10.90 .6 & 2233 & 44 & 2781 & 71 & -0.98 \\
\hline $7-2 \mathrm{C}$ & $7.2 \mathrm{C}$ & 1674 & 0.094208 & 0.001835 & 0.281689 & 0.000020 & -3.10 .7 & 2242 & 56 & 2558 & 87 & -0.94 \\
\hline $8-2 \mathrm{C}$ & $8.2 \mathrm{C}$ & 2015 & 0.029745 & 0.000626 & 0.281675 & 0.000018 & 5.30 .6 & 2191 & 49 & 2298 & 79 & -0.98 \\
\hline $9-1 \mathrm{C}$ & $9.1 \mathrm{C}$ & 1968 & 0.058211 & 0.001429 & 0.281649 & 0.000024 & 2.30 .9 & 2273 & 67 & 2448 & 105 & -0.96 \\
\hline $10-1 \mathrm{C}$ & $10.1 \mathrm{C}$ & 1848 & 0.078311 & 0.001670 & 0.281721 & 0.000018 & 1.90 .6 & 2187 & 50 & 2378 & 77 & -0.95 \\
\hline $11-1 \mathrm{C}$ & $11.1 \mathrm{C}$ & 1890 & 0.082093 & 0.001996 & 0.281673 & 0.000021 & 0.70 .7 & 2273 & 58 & 2484 & 90 & -0.94 \\
\hline $12-1 \mathrm{C}$ & $12.1 \mathrm{C}$ & 1856 & 0.071574 & 0.001456 & 0.281714 & 0.000018 & 2.10 .7 & 2184 & 51 & 2371 & 80 & -0.96 \\
\hline $13-1 \mathrm{C}$ & $13.1 \mathrm{C}$ & 1995 & 0.051751 & 0.001207 & 0.281638 & 0.000019 & 2.80 .7 & 2275 & 53 & 2438 & 84 & -0.96 \\
\hline 14-1RC & 14.1RC & 1612 & 0.024642 & 0.000639 & 0.281636 & 0.000021 & $\begin{array}{ll}-5 & 0.8\end{array}$ & 2244 & 58 & 2628 & 94 & -0.98 \\
\hline $18-1 \mathrm{C}$ & $18.1 \mathrm{C}$ & 1924 & 0.066004 & 0.001744 & 0.281494 & 0.000025 & -4.60 .9 & 2510 & 70 & 2837 & 110 & -0.95 \\
\hline
\end{tabular}

Note: D in samples QT1-12b and LT1-11 means detrital core of zircon, C in samples S2-5, H3-7 and QS1-18 means central domain of zircon, with non-genetic implication, RC and R in all samples mean recrystallized zircon domain and zircon rim, respectively.

values of -11.0 to 3.3 and Hf crustal model ages of 2418 to 3235 Ma and thus generally have slightly older Hf model ages than the magmatic core domains.

\section{PROTOLITH AGES OF THE METAMORPHIC ROCKS}

Based on the zircon data, two age groups are recognized. Only one sample (S2-5) contains early Paleoproterozoic zircons and this was obtained from the northern margin of the Ordos Basin (fig. 2). In this sample, the "core" zircon domains are all discordant, with the oldest grain recording a minimum ${ }^{207} \mathrm{~Pb} /{ }^{206} \mathrm{~Pb}$ age of $2544 \pm 15$ Ma (22.1C), but with a strong discordance The age of the dark rims, although similarly discordant, suggests that the timing of metamorphism was $>2.28 \mathrm{Ga}$. This implies that a metamorphic event occurred during the early Paleoproterozoic, therefore limiting the formation age of the rock to $>2.28 \mathrm{Ga}$. There are thus two possibilities for the formation age of sample S2-5. If it is an orthogneiss, its protolith formed in the late Neoarchean to earliest Paleoproterozoic. Alternatively, if it is a paragneiss, as we suggest, deposition of the protolith was most probably between $\sim 2.5$ and $2.4 \mathrm{Ga}$. Early Paleoproterozoic metasedimentary rocks have been identified in the Daqingshan area (Wan and others, 2009; Dong and others, 2013), about $80 \mathrm{~km}$ to the north of the sample location, suggesting that the Daqingshan and northern Ordos were part of the same basement terrane at that time. 

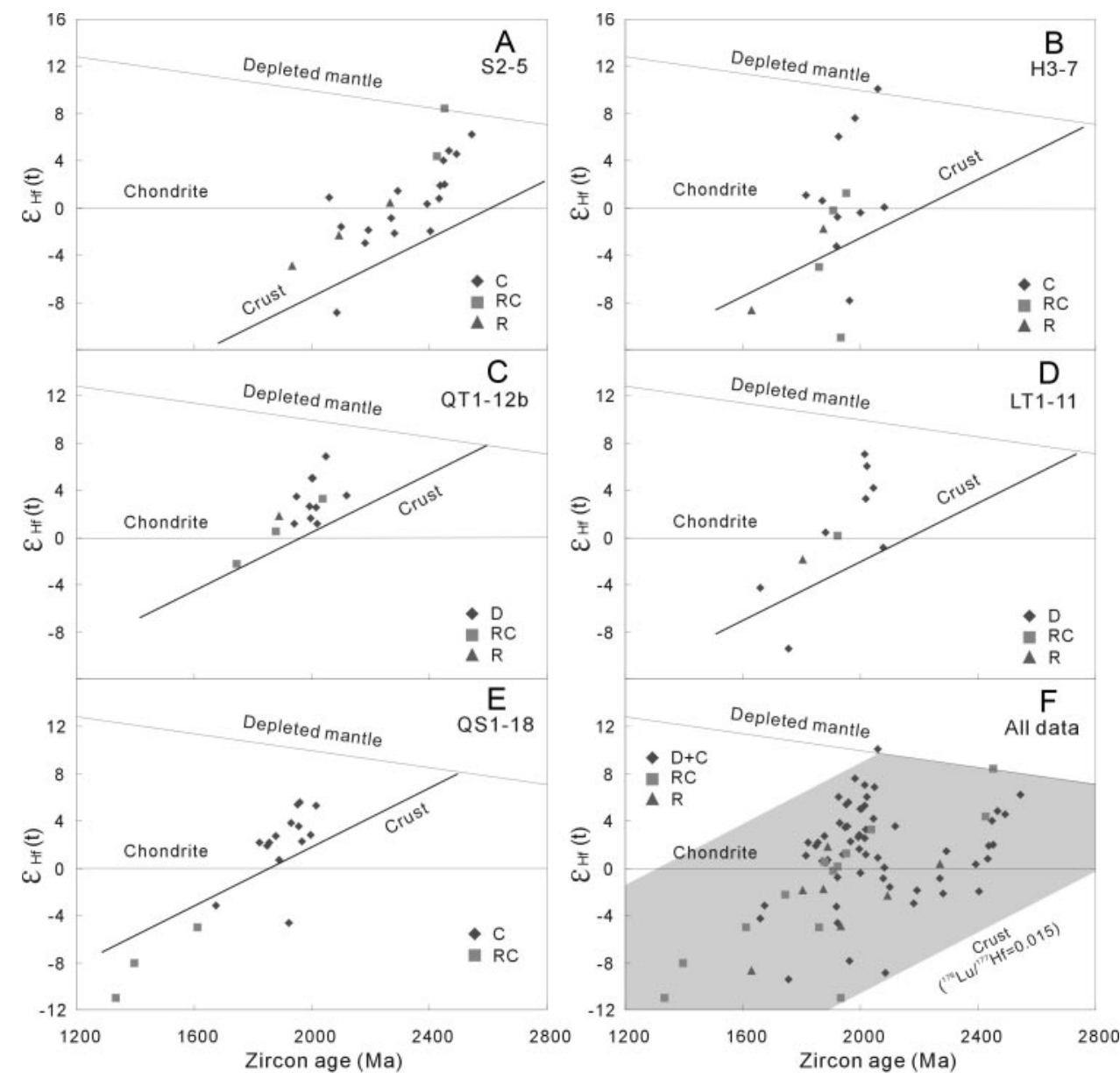

Fig. 6. Diagram of $\varepsilon_{\mathrm{Hf}}(\mathrm{t})$ vs. U-Pb age for zircons from Paleoproterozoic rocks obtained from drill cores beneath the Ordos Basin. (A) Biotite K-feldspar gneiss (S2-5); (B) banded two-mica K-feldspar gneiss (H3-7); (C) garnet-sillimanite-biotite gneiss (QT1-12b); (D) graphite-bearing two-mica two-feldspar gneiss (LT1-11);

(E) altered mica quartz-feldspar gneiss (QS1-18); (F) all data. D, C, RC and R are same as in figure 4.

The other age group includes all remaining samples (H3-7, QT1-12b, LT1-11, QS1-18) that cover a wide area beneath the Ordos Basin (fig. 2). As mentioned above, samples QT1-12b and LT1-11 are of metasedimentary origin because they contain garnet, sillimanite and graphite. However, samples H3-7 and QS1-18 are of uncertain origin (orthogneiss or paragneiss) although we favor a sedimentary protolith, as discussed above. Most zircon cores in all these samples have ${ }^{207} \mathrm{~Pb} /{ }^{206} \mathrm{~Pb}$ ages of 2.1 to 2.0 Ga, making them significantly younger than those in sample S2-5. The least altered cores with magmatic oscillatory zoning indicate that they were derived from a granitoid terrane of possible middle Paleoproterozoic age (in the case of paragneiss), or that they represent the magmatic age of the protolith (in the case of orthogneiss). Only imprecise metamorphic ages of $1947 \pm 74$ and $1882 \pm 45$ Ma were obtained from samples in this study because of strong lead loss from the metamorphic domains. However, such ages have previously been reported from adjacent parts of the NCC, including Daqingshan to the north (Wan and others, 2006, 2009, 2013; Dong and 
others, 2012, 2013; Ma and others, 2012a). A 1950 Ma high-grade metamorphic event was recorded by Yin and others (2009) from the Qianlishan Complex in the westernmost part of the Khondalite Belt (fig. 1), and $\sim 1.8 \mathrm{Ga}$ metamorphism was reported from both the eastern part of the Khondalite Belt near its contact with the TNCO (Zhao and others, 2010) and widely from the interior of the TNCO (see Zhao and others, 2005).

Therefore, an important conclusion is that Paleoproterozoic metasedimentary rocks are widely distributed across the Ordos basement and underwent strong deformation and metamorphism at the end of the Paleoproterozoic. In this respect these are similar, in both rock association and metamorphic history, to rocks surrounding the Ordos Basin, including the Lüliang, Jining, Daqingshan and Qianlishan complexes (Wan and others, 2006, 2009; Dong and others, 2007, 2012; Xia and others, 2009; Yin and others, 2009; Zhou and Geng, 2009; Dan and others, 2011; Guo and others, 2011).

These late Paleoproterozoic supracrustal rocks and others exposed elsewhere in the NCC, including the Daqingshan area to the north of the Ordos Basin (Wan and others, 2009; Dong and others, 2012), predominantly consist of continent-derived material. No unconformity between the late Paleoproterozoic supracrustal rocks and Archean to early Paleoproterozoic crystalline basement has so far been identified any where in the NCC, and the relationships are tectonic at all localities where the boundary can be observed (Guo and others, 2005; Wan and others, 2009). It is possible, however, that at least some of the late Paleoproterozoic rocks were deposited unconformably on older basement as suggested by the occurrence of Archean and early Paleoproterozoic crustal fragments in the Khondalite Belt (Zhang and others, 2009; Wan and others, 2009; Ma and others, 2012b). In some areas such as Huai' an and eastern Shandong, furthermore, the Archean rocks underwent the same late Paleoproterozoic high-grade metamorphism as nearby late Paleoproterozoic rocks (Guo and others, 2005; Wan and others, 2006). We therefore infer that late Paleoproterozoic supracrustal rocks were once more extensively distributed than is apparent today. Combined with the above observations, identification of late Paleoproterozoic sedimentary rocks $(\sim 2.0-1.95 \mathrm{Ga})$ in the basement of the Ordos Basin indicates that such supracrustal sequences were widely distributed, with the total area extending across almost half of the NCC.

Ca. 2.5 Ga AND 2.1 TO 2.0 Ga MAGMATISM IN THE ORDOS BLOCK AND ADJACENT AREAS

The oscillatory-zoned magmatic zircons in sample S2-5 with ages between 2.55 and $2.50 \mathrm{Ga}$ are consistent with the conclusion that a late Neoarchean tectono-magmatic event occurred widely throughout the NCC (Wan and others, 2011; Zhao and Zhai, 2013, and references therein). These zircons also show variations in their Hf isotopic compositions with $\varepsilon_{\mathrm{Hf}}(2.5 \mathrm{Ga})$ values ranging from below chondritic to the depleted mantle line (fig. 6A). This suggests that many of these grains were derived from older continental material, with some recording the addition of a juvenile component. The Hf crustal model ages range from 3.2 to $2.6 \mathrm{Ga}$ (table 3 ), with an average of $\sim 2.8 \mathrm{Ga}$, broadly consistent with the Hf crustal model age of similar rocks across the entire NCC (Geng and others, 2012).

All late or middle-late Paleoproterozoic samples (H3-7, QT1-12b, LT1-11, QS1-18) show a similar zircon age distribution (fig. 7A). Abundant 2.1 to 2.0 Ga zircon cores with magmatic structures indicate that widespread magmatism occurred during the middle Paleoproterozoic (2.2-2.0 Ga) in the Ordos Block and adjacent areas (Wan and others, 2006; Dong and others, 2012; Santosh and others, 2013). The sedimentary protoliths were mainly derived from magmatic sources of this age. This is similar to the late Paleoproterozoic khondalites farther north in the Khondalite Belt, which contain considerably more middle Paleoproterozoic detrital zircons than late Neoarchean grains (Dong and others, 2012; Wan and others, 2006, 2009). Several geological units 

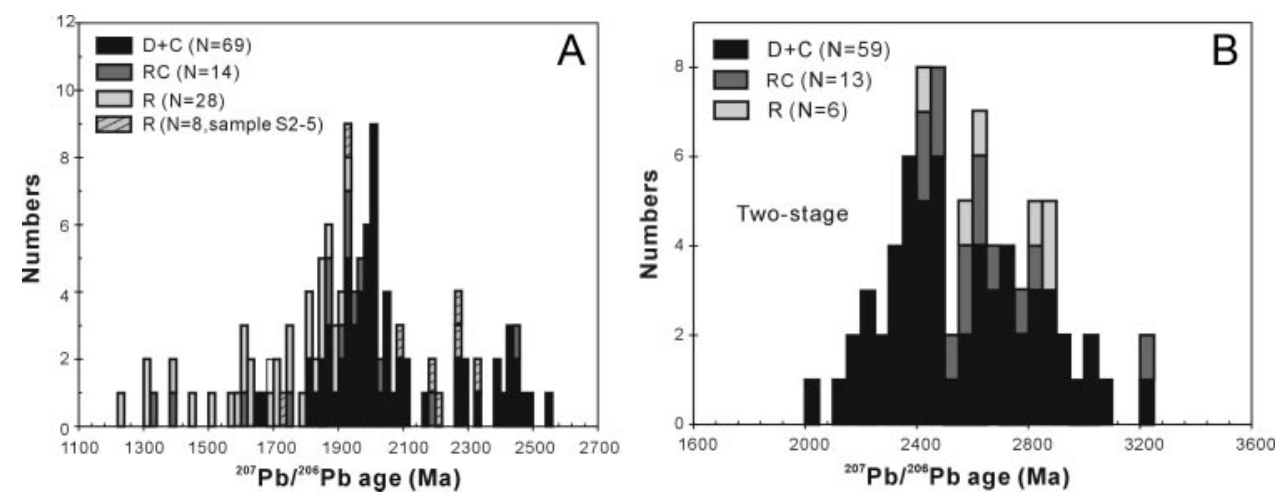

Fig. 7. ${ }^{207} \mathrm{~Pb} /{ }^{206} \mathrm{~Pb}$ age histogram (A) and Hf crustal model age histogram (B) of zircons from samples obtained from drill cores beneath the Ordos Basin. D, C, RC and R are as in figure 4.

with an age of 2.3 to $2.0 \mathrm{Ga}$, including supracrustal rocks and mafic to felsic intrusive rocks, have been identified in the NCC, such as in Alax, Helanshan, Daqingshan, Zhongtiaoshan, Lüliang, Wutai, Hengshan, Fuping, Liaoning and eastern Shandong (fig. 1) (Wan and others, 2011; Zhao and Zhai, 2013, and references therein).

Zircon cores from all late (or middle-late) Paleoproterozoic samples show a large variation in $\varepsilon_{\mathrm{Hf}}(\mathrm{t})$ from -9.1 to 10.1 (figs. $6 \mathrm{~B}, 6 \mathrm{C}, 6 \mathrm{D}, 6 \mathrm{E}$, and $6 \mathrm{~F}$ ), suggesting that both juvenile components and crustal sources played important roles in their crystallization during the middle Paleoproterozoic. The spread of $\varepsilon_{\mathrm{Hf}}(\mathrm{t})$ values at $\sim 2.0 \mathrm{Ga}$ (fig. $6 \mathrm{~F}$ ) from chondrite to the depleted mantle line possibly signities a major magmatic event at this time, which correlates with abundant oscillatorily zoned magmatic zircons of this age both here and elsewhere in the NCC. It is also evident that at least some of the middle Paleoproterozoic magmatic rocks were derived from Neoarchean continental crust, a conclusion also indicated from the study of detrital zircons in late Paleoproterozoic metasedimentary rocks (khondalites) in other areas of the NCC (Xia and others, 2006, 2008; Wan and others, 2009).

Two or three age peaks are evident when all data are plotted on a Hf isotope crustal model age histogram (fig. 7B). The model age peaks occur at $\sim 2.4, \sim 2.65$, and $\sim 2.85 \mathrm{Ga}$. Importantly, although some zircons show a cluster around $2.7 \mathrm{Ga}$, which is characteristic of the NCC in general, many zircons from beneath the Ordos Basin have Hf model ages of $\sim 2.4 \mathrm{Ga}$, broadly similar to the $\sim 2.3 \mathrm{Ga}$ values recorded from khondalitic rocks immediately to the north (Geng and others, 2012). However, it is important to point out that the Hf model ages are estimates, and there are considerable errors because of the uncertainty in the true ${ }^{176} \mathrm{Lu} /{ }^{177} \mathrm{Hf}$ ratios of the protoliths of the dated samples.

GEOLOGICAL IMPLICATION FOR THE PALEOPROTEROZOIC EVOLUTION OF THE NCG

We analyzed drill-core samples from basement rocks beneath the Ordos Basin, and these, as well as similar samples studied by $\mathrm{Hu}$ and others (2012), yielded Paleoproterozoic rock-formation ages. Six samples contain only middle Paleoproterozoic detrital zircons, whereas one early Paleoproterozoic (or Neoarchean) sample (S2-5) contains $\sim 2.5 \mathrm{Ga}$ zircons. In this respect, these are similar to rocks exposed in the Khondalite Belt to the north of the Ordos Basin (figs. 1 and 2), and it is likely that these zircons and their hosts were either derived from, or form part of, the Khondalite Belt. On the basis of this study, and given the wide distribution of samples, we find no evidence of Archean rocks, except possibly for sample S2-5, whose protolith may have 
formed at the end of the Neoarchean. Furthermore, there is no evidence that Archean source terranes were exposed in the area during deposition of the late Paleoproterozoic sediments.

The seven samples available for study are widely distributed across the area (fig. 2) and show an absence or rarity of Archean zircons. It therefore seems likely that the upper crust of the Ordos Block is composed of Paleoproterozoic rocks. However, given its thick sub-continental lithosphere, it is possible that Archean rocks may still be present in the lower crust. It is also evident that the widely distributed late (or middle-late) Paleoproterozoic rocks underwent strong metamorphism and deformation at the end of the Paleoproterozoic, indicating that the Ordos basement was involved in a major late Paleoproterozoic tectono-thermal event. This has important implications for the assumed collisional history of the NCC. The two most popular models for the formation of the NCC involve collision of the Eastern and Western Blocks along a central orogenic belt, as discussed in the introduction. It has been assumed in all models that the Western Bock was predominantly of Archean age, represented by the basement below the Ordos Basin. Our results imply that neither of these models is correct. In addition, our conclusions bring into question the concept of a Trans-North China Orogen (TNCO), as proposed by Zhao and others (2000) that was supposed to have resulted from the collision, at about $1.85 \mathrm{Ga}$ ago, of two Archean blocks. The consistent clockwise $\mathrm{P} / \mathrm{T}$ paths recorded in metamorphic rocks of the TNCO (Zhao and others, 2000) support the view that the high-grade assemblages in this belt may indeed have been the result of collisional processes, but our results suggest that this event involved a Paleoproterozoic Western (Ordos) Block.

\section{CONCLUSIONS}

One sample (S2-5) from beneath the northern margin of the Ordos Basin is an early Paleoproterozoic metasedimentary or late Neoarchean magmatic rock with an early Paleoproterozoic metamorphic history. Hf-in-zircon isotopes suggest that it was derived from older continental material with the possible addition of a juvenile component.

Six samples (including those of $\mathrm{Hu}$ and others, 2012) are late Paleoproterozoic metasediments, although some with uncertain protoliths may possibly be middle Paleoproterozoic magmatic rocks. Hf-in-zircon isotopes imply that recycling of Neoarchean crustal material played an important role in their crystallization during the middle Paleoproterozoic.

Late Paleoproterozoic metasedimentary rocks are widely distributed in the Ordos basement and were involved in a major late Paleoproterozoic tectono-thermal event that is also recorded in other parts of the NCC. This questions previous models in which the basement to the Ordos Basin (the Ordos Block) was considered to be an Archean block that collided with other Archean blocks to form the NCC.

ACKNOWLEDGMENTS

We thank Yuhai Zhang and Jianhui Liu for help with SHRIMP data collection, Chun Yang for making the zircon mounts and Ning Li for CL imaging. We are grateful to Qihan Shen, Minggou Zhai, Chonghui Yang, Jinghui Guo, Shuwen Liu, Gouchun Zhao and Allen Nutman for discussions on these findings. We thank M. Santosh and Allen Nutman for thoughtful reviews. This project was supported by grants from the Ministry of Land and Resources of the Peoples' Republic of China (1212011120151, 1212011121062) and the Major State Basic Research Development Program of China (973 Program) (2012CB416600). 


\section{REFERENCES}

Bai, J., 1993, The Precambrian Geology and Pb-Zn Mineralization in the Northern Margin of North China Platform: Beijing, Geological Publishing House (in Chinese with English abstract).

Black, L. P., Kamo, S. L., Allen, C. M., Aleinikoff, J. K., Davis, D. W., Korsch, R. J., and Foudoulis, C., 2003, TEMORA 1: a new zircon standard for Phanerozoic U-Pb geochronology: Chemical Geology, v. 200, n. 1-2, p. 155-170, http://dx.doi.org/10.1016/S0009-2541(03)00165-7

Bouvier, A., Vervoort, J. D., and Patchett, P. J., 2008, The Lu-Hf and Sm-Nd isotopic composition of CHUR: Constraints from unequilibrated chondrites and implications for the bulk composition of terrestrial planets: Earth and Planetary Science Letters, v. 273, n. 1-2, p. 48-57, http://dx.doi.org/10.1016/ j.epsl.2008.06.010

Changqing Oilfield Company of PetroChina (COCP), 2011, Introduction on drilling cores relating to Precambrian basement in the Ordos Basin: Unpublished ms, 20 p.

Chen, L., 2010, Concordant structural variations from the surface to the base of the upper mantle in the North China Craton and its tectonic implications: Lithos, v. 120, n. 1-2, p. 96-115, http://dx.doi.org/ $10.1016 / \mathrm{j}$. lithos.2009.12.007

Chen, L., Cheng, C., and Wei, Z., 2009, Seismic evidence for significant lateral variations in lithospheric thickness beneath the central and western North China Craton: Earth and Planetary Science Letters, v. 286, n. 1-2, p. 171-183. http://dx.doi.org/10.1016/j.epsl.2009.06.022

Chu, N. C., Taylor, R. N., Chavagnac, V., Nesbitt, R. W., Boella, R. M., Milton, J. A., German, C. R., Bayon, G., and Burton, K., 2002, Hf isotope ratio analysis using multi-collector inductively coupled plasma mass spectrometry: an evaluation of isobaric interference corrections: Journal of Analytical Atomic Spectrometry, v. 17, p. 1567-1574, http://dx.doi.org/10.1039/b206707b

Cumming, G. L., and Richards, J. R., 1975, Ore lead isotope ratios in a continuously changing earth: Earth and Planetary Science Letters, v. 28, n. 2, p. 155-171, http://dx.doi.org/10.1016/0012-821X(75)90223-X

Dan, W., Li, X.-H., Guo, J. H., Liu, Y., and Wang, X.-C., 2011, Integrated in situ zircon U-Pb age and Hf-O isotopes for the Helanshan khondalites in North China Craton: juvenile crustal materials deposited in active or passive continental margin: Precambrian Research, http://dx.doi.org/10.1016/j.precamres. 2011.07.016

Diwu, C. R., Sun Y., Zhang H., Wang Q., Guo A. L., and Fan, L. G., 2011, Episodic tectonothermal events of the western North China Craton and North Qinling Orogenic Belt in central China: Constraints from detrital zircon U-Pb ages: Journal of Asian Earth Sciences, v. 47, p. 107-122, http://dx.doi.org/10.1016/ j.jseaes.2011.07.012

Dong, C. Y., Liu, D. Y., Li, J. J., Wang, Y. S., Zhou, H. Y., Li, C. D., Yang, Y. H., and Xie, L. W., 2007, Palaeoproterozoic Khondalite Belt in the western North China Craton: New evidence from SHRIMP dating and $\mathrm{Hf}$ isotope composition of zircons from metamorphic rocks in the Bayan U-Helan mountain area: Chinese Science Bulletin, v. 52, n. 21, p. 2984-2994, http://dx.doi.org/10.1007/s11434-0070404-9

Dong, C. Y., Wan, Y. S., Xu, Z. Y., Liu, D. Y., Yang, Z. S., Ma, M. Z., and Xie, H. Q., 2012, Late Paleoproterozoic kondalites in the Daqingshan area, North China Craton: SHRIMP zircon U-Pb dating: Science China Earth Sciences, http://dx.doi.org/10.1007/s11430-012-4459-3

Dong, C. Y., Wan, Y. S., Wilde, S. A., Xu, Z. Y., Ma, M. Z., Xie, H. Q., and Liu, D. Y., 2013, Earliest Paleoproterozoic supracrustal rocks in the North China Craton recognized from the Daqingshan area of the Khondalite Belt: Constraints on craton evolution: Gondwana Research, http://dx.doi.org/ 10.1016/j.gr.2013.05.021

Elhlou, S., Belousova, E., Griffin, W. L., Pearson, N. J., and O'Reilly, S. Y., 2006, Trace element and isotopic composition of GJ-red zircon standard by laser ablation: Geochimica et Cosmochimica Acta, v. 70, n. 18, supplement, p. A158, http://dx.doi.org/10.1016/j.gca.2006.06.1383

Faure, M., Lin, W., Monié, P., and Bruguier, O., 2004, Palaeoproterozoic arc magmatism and collision in Liaodong Peninsula (north-east China): Terra Nova, v. 16, n. 2, p. 75-80, http://dx.doi.org/10.1111/ j.1365-3121.2004.00533.x

Geng, Y. S., Wan, Y. S., Shen, Q. H., Li, H. M., and Zhang, R. X., 2000, Chronological framework of the early Precambrian important events in the Lüliang area, Shanxi Province: Acta Geologica Sinica, v. 74, p. 216-223 (in Chinese with English abstract).

Geng, Y. S., Yang, C. H., Song, B., and Wan, Y. S., 2004, Post-orogenic granites with an age of 1800 Ma in the Lüliang area, North China Craton: Constraints from geochronology and geochemistry: Geological Journal of China Universities, v. 10, p. 477-487 (in Chinese with English abstract).

Geng, Y. S., Yang, C. H., and Wan, Y. S., 2006, The evolution of Paleoproterozoic granites in Lüliang area, North China Craton: Constraint from isotopic geochronology: Acta Petrologica Sinica, v. 22, p. 305-314 (in Chinese with English abstract).

Geng, Y. S., Du, L. L., and Ren, L. D., 2012, Growth and reworking of the early Precambrian continental crust in the North China Craton Constraints from zircon Hf isotopes: Gondwana Research, v. 2, p. 517-529, http:/ /dx.doi.org/10.1016/j.gr.2011.07.006

Griffin, W. L., Pearson, N. J., Belousova, E., Jackson, S. E., O’Reilly, S. Y., van Achterberg, E., and Shee, S. R., 2000, The Hf-isotope composition of cratonic mantle: LAM-MC-ICPMS analysis of zircon megacrysts in kimberlites: Geochimica et Cosmochimica Acta, v. 64, n. 2-3, p. 133-147, http://dx.doi.org/10.1016/ S0016-7037(99)00343-9

Guo, J. H., Sun, M., Chen, F. K., and Zhai, M. G., 2005, Sm-Nd and SHRIMP U-Pb zircon geochronology of high-pressure granulites in the Sanggan area, North China Craton: timing of Paleoproterozoic continental collision: Journal of Asian Earth Sciences, v. 24, n. 5, p. 629-642, http://dx.doi.org/10.1016/ j.jseaes.2004.01.017

Guo, J. H., Peng, P., Chen, Y., Jiao, S. J., and Windley, B. F., 2011, UHT sapphirine granulite metamorphism 
at 1.93-1.92 Ga caused by gabbronorite intrusions: Implications for tectonic evolution of the northern margin of the North China Craton: Precambrian Research, v. 222-223, p. 124-142, http:/ /dx.doi.org/ 10.1016/j.precamres.2011.07.020

He, G. P., and Ye, H. W., 1998, Two type of Early Proterozoic metamorphism in the eastern Liaoning to southern Jilin and their tectonic implication: Acta Petrologica Sinica, v. 14, p. 152-162.

Hou, K. J., Li, Y. H., Zou, T. R., Qu, X. M., Shi, Y. R., and Xie, G. Q., 2007, Laser ablation-MC-ICP-MS technique for Hf isotope microanalysis of zircon and its geological applications: Acta Petrologica Sinica, v. 23, p. 2595-2604 (in Chinese with English abstract).

Hu, J. M., Liu, X. S., Li, Z. H., Zhao, Y., Zhang, S. H., Liu, X. C., Qu, H. J., and Chen, H., 2012, SHRIMP U-Pb zircon dating of the Ordos Basin basement and its tectonic significance: Chinese Science Bulletin, v. 58, n. 1, p. 118-127, http://dx.doi.org/10.1007/s11434-012-5274-0

Hu, N. G., Yang, J. X., Wang, Z. B., Wang, T., and Li, W. P., 1994, The composition and evolution of complex in Helanshan: Xi'an, Xi'an Map Publishing House, 59 p. (in Chinese).

$\mathrm{Hu}, \mathrm{S}$., He, L., and Wang, J., 2000, Heat flow in the continental area of China: a new data set: Earth and Planetary Science Letters, v. 179, n. 2, p. 407-419, http:/ /dx.doi.org/10.1016/S0012-821X(00)00126-6

Jiang, M. L., Ai, Y. S., Chen, L., and Yang, Y. J., 2013, Local modification of the lithosphere beneath the central and western North China Craton: 3-D constraints from Rayleigh wave tomography: Gondwana Research, http://dx.doi.org/10.1016/j.gr.2012.06.018

Jiang, Z. S., Wang, G. D., Xiao, L. L., Diwu, C. R., Lu, J. S., and Wu, C. M., 2011, Paleoproterozoic metamorphic P-T-t path and tectonic significance of the Luoning metamorphic complex at the southern terminal of the Trans-North China Orogen, Henan Province: Acta Petrologica Sinica, v. 27, n. 12, p. 3701-3717.

Kröner, A., Wilde, S. A., Li, J. H., and Wang, K. Y., 2005, Age and evolution of a late Archean to Paleoproterozoic upper to lower crustal section in the Wutaishan/Hengshan/Fuping terrain of northern China: Journal of Asian Earth Science, v. 24, n. 5, p. 577-595, http://dx.doi.org/10.1016/ j.jseaes.2004.01.001

Kröner, A., Wilde, S. A., Zhao, G. C., O'Brien, P. J., Sun, M., Liu, D. Y., Wan, Y. S., Liu, S. W., and Guo, J. H., 2006, Zircon geochronology and metamorphic evolution of mafic dykes in the Hengshan Complex of northern China: Evidence for late Palaeoproterozoic extension and subsequent high-pressure metamorphism in the North China Craton: Precambrian Research, v. 146, n. 2, p. 45-67, http://dx.doi.org/ 10.1016/j.precamres.2006.01.008

Kusky, T. M., and Li, J. H., 2003, Paleoproterozoic tectonic evolution of the North China Craton: Journal of Asian Earth Science, v. 22, n. 4, p. 383-397, http://dx.doi.org/10.1016/S1367-9120(03)00071-3

Kusky, T. M., Li, J. H., and Santosh, M., 2007, The Paleoproterozoic North Hebei Orogen: North China Craton's collisional suture with the Columbia supercontinent: Gondwana Research, v. 12, n. 1-2, p. 4-28, http:/ /dx.doi.org/10.1016/j.gr.2006.11.012

Li, J. H., Kusky, T. M., and Huang, X., 2002, Archean podiform chromitites and mantle tectonites in ophiolitic mélange, North China Craton: A record of early oceanic mantle processes: GSA Today, v. 12, n. 7, p. 4-11, http://dx.doi.org/10.1130/1052-5173(2002)012〈0004:APCAMT $\rangle 2.0 . C O ; 2$

Li, X.-P., Yang, Z. Y., Zhao, G. C., Grapes, G., and Guo, J. H., 2010, Geochronology of khondaliteseries rocks of the Jining Complex: confirmation of depositional age and tectonometamorphic evolution of the North China Craton: International Geology Review, v. 53, n. 10, p. 1194-1211, http://dx.doi.org/ $10.1080 / 00206810903548984$

Liu, S. J., Dong, C. Y., Xu, Z. Y., Santosh, M., Ma, M. Z., Xie, H. Q., Liu, D. Y., and Wan, Y. S., 2012, Paleoproterozoic episodic magmatism and high-grade metamorphism in the North China Craton: Evidence from SHRIMP zircon dating of magmatic suites from the Daqingshan area: Geological Journal, http://dx.doi.org/10.1002/gj.2453

Liu, S. W., Li, Q. G., Liu, C. H., Lü, Y. J., and Zhang, F., 2009, Guandishan Granitoids of the Paleoproterozoic Lüliang Metamorphic Complex in the Trans-North China Orogen: SHRIMP Zircon Ages, Petrogenesis and Tectonic Implications: Acta Geologica Sinica, v. 83, n. 3, p. 580-602, http://dx.doi.org/10.1111/ j.1755-6724.2009.00051.x

Liu, S. W., Zhang, J., Li, Q. G., Zhang, L. F., Wang, W., and Yang, P. T., 2011, Geochemistry and U-Pb zircon ages of metamorphic volcanic rocks of the Paleoproterozoic Lüliang Complex and constraints on the evolution of the Trans-North China Orogen, North China Craton: Precambrian Research, v. 222-223, p. 173-190, http://dx.doi.org/10.1016/j.precamres.2011.07.006

Lu, L. Z., Xu, X. C., and Liu, F. L., 1996, Early Precambrian Khondalite Series of North China: Changchun, Changchun Publishing House, 272 p. (in Chinese).

Ludwig, K. R., 2001, Squid 1.02: a user's manual: Berkeley Geochronology Centre, Special Publication 2 , 19 p.

2003, User's Manual for Isoplot 3.00, a Geochronological Toolkit for Microsoft Excel. Berkeley Geochronology Center, 70 p.

Luo, Y., Sun, M., Zhao, G. C., Li, S. Z., Xu, P., Ye, K., and Xia, X. P., 2004, LA-ICP-MS U-Pb zircon ages of the Liaohe Group in the Eastern Block of the North China Craton: constraints on the evolution of the Jiao-Liao-Ji Belt: Precambrian Research, v. 134, n. 3-4, p. 349-371, http://dx.doi.org/10.1016/ j.precamres.2004.07.002

Ma, M. Z., Wan, Y. S., Xu, Z. Y., Liu, S. J., Xie, H. Q., Dong, C. Y., and Liu, D. Y., 2012a, Late Paleoproterozoic K-feldspar pegmatite vein in the Daqingshan area, North China Craton: SHRIMP age and Hf composition of zircons: Geological Bulletin of China, v. 31, p. 825-833 (in Chinese with English abstract).

Ma, M. Z., Wan, Y. S., Santosh, M., Xu, Z. Y., Xie, H. Q., Dong, C. Y., and Liu, D. Y., 2012b, Decoding multiple tectonothermal events in zircons from single rock samples: SHRIMP zircon U-Pb data from the late Neoarchean rocks of Daqingshan, North China Craton: Gondwana Research, v. 22, n. 3-4, p. 810-827, http://dx.doi.org/10.1016/j.gr.2012.02.020 
Santosh, M., 2010, Assembling North China Craton within the Columbia supercontinent: The role of double-sided subduction: Precambrian Research, v. 178, n. 1-4, p. 149-167, http://dx.doi.org/10.1016/ j.precamres.2010.02.003

Santosh, M., Sajeev, K., and Li, J. H., 2006, Extreme crustal metamorphism during Columbia supercontinent assembly: evidence from North China Craton: Gondwana Research, v. 10, n. 3-4, p. 256-266, http:// dx.doi.org/10.1016/j.gr.2006.06.005

Santosh, M., Wilde, S. A., and Li, J. H., 2007, Timing of Palaeoproterozoic ultrahigh-temperature metamorphism in the North China Craton: evidence from SHRIMP U-Pb zircon geochronology: Precambrian Research, v. 159, n. 3-4, p. 178-196, http://dx.doi.org/10.1016/j.precamres.2007.06.006

Santosh, M., Wan, Y. S., Liu, D. Y., Dong, C. Y., and Li, J. H., 2009, Anatomy of zircons from an ultrahot orogen: the amalgamation of the North China Craton within the Columbia supercontinent: Journal of Geology, v. 117, n. 4, p. 429-443, http://dx.doi.org/10.1086/598949

Santosh, M., Liu, D. Y., Shi, Y. R., and Liu, S. J., 2013, Paleoproterozoic accretionary orogenesis in the North China Craton: a SHRIMP study: Precambrian Research, v. 227, p. 29-54, http://dx.doi.org/10.1016/ j.precamres.2011.11.004

Shen, Q. H., Zhang, Y. F., Gao, J. F., and Wang, P., 1990, Study on Archaean Metamorphic Rocks in midsouthern Nei Mongol of China: Beijing, Geological Publishing House, 192 p. (in Chinese).

Söderlund, U., Patchett, J. P., Vervoort, J. D., and Isachsen, C. E., 2004, The ${ }^{176}$ Lu decay constant determined by Lu-Hf and U-Pb isotope systematics of Precambrian mafic intrusions: Earth and Planetary Science Letters, v. 219, n. 3-4, p. 311-324, http://dx.doi.org/10.1016/S0012-821X(04)00012-3

Song, L. J., Chen, J. L., Zhang, Y. L., Liu, C. Y., Wu, C. L., and Zhang, X. H., 2010, U-Pb chronological characteristics of late Triassic sediment in southwestern Ordos and it's tectonic significance: Acta Geologica Sinica, v. 84, p. 370-386.

Tsunogae, T., Liu, S. J., Santosh, M., Shimizu, H., and Li, J. H., 2011, Ultrahigh-temperature metamorphism in Daqingshan, Inner Mongolia Suture Zone, North China Craton: Gondwana Research, v. 20, n. 1 , p. 36-47, http://dx.doi.org/10.1016/j.gr.2011.03.001

Wan, Y. S., Song, B., Liu, D. Y., Wilde, S. A., Wu, J. S., Shi, Y. R., Yin, X. Y., and Zhou, H. Y., 2006, SHRIMP $\mathrm{U}-\mathrm{Pb}$ zircon geochronology of Palaeoproterozoic metasedimentary rocks in the North China Craton: Evidence for a major Late Palaeoproterozoic tectonothermal event: Precambrian Research, v. 149, n. 3-4, p. 249-271, http://dx.doi.org/10.1016/j.precamres.2006.06.006

Wan, Y. S., Liu, D. Y., Xu, Z. Y., Dong, C. Y., Wang, Z. J., Zhou, H. Y., Yang, Z. S., Liu, Z. H., and Wu, J. S., 2008, Paleoproterozoic crustally derived carbonate-rich magmatic rocks from the Daqinshan area, North China Craton: Geological, petrographical, geochronological and geochemical (Hf, Nd, O and $\mathrm{C}$ ) evidence: American Journal of Science, v. 308, n. 3, p. 351-378, http://dx.doi.org/10.2475/03.2008.07

Wan, Y. S., Liu, D. Y., Dong, C. Y., Xu, Z. Y., Wang, Z. J., Wilde, S. A., Yang, Y. H., Liu, Z. H., and Zhou, H. Y., 2009, The Precambrian Khondalite Belt in the Daqingshan area, North China Craton: evidence for multiple metamorphic events in the Palaeoproterozoic, in Reddy, S. M., Mazumder, R., Evans, D. A. D., and Collins, A. S., editors, Palaeoproterozoic Supercontinents and Global Evolution: Geological Society, London, Special Publications, v. 323, p. 73-97, http://dx.doi.org/10.1144/SP323.4

Wan, Y. S., Liu, D. Y., Wang, W., Song, T. R., Kröner, A., Dong, C. Y., Zhou, H. Y., and Yin, X. Y., 2011, Provenance of Meso- to Neoproterozoic cover sediments at the Ming Tombs, Beijing, North China Craton: An integrated study of U-Pb dating and Hf isotopic measurement of detrital zircons and whole-rock geochemistry: Gondwana Research, v. 20, n. 1, p. 219-242, http://dx.doi.org/10.1016/ j.gr.2011.02.009

Wan, Y. S., Xu, Z. Y., Dong, C. Y., Nutman, A., Ma, M. Z., Xie, H. Q., Liu, S. J., Liu, D. Y., Wang, H. C., and Cu, H., 2013, Episodic Paleoproterozoic $(\sim 2.45, \sim 1.95$ and $\sim 1.85 \mathrm{Ga})$ mafic magmatism and associated high temperature metamorphism in the Daqingshan area, North China Craton: SHRIMP zircon U-Pb dating and whole-rock geochemistry: Precambrian Research, v. 224, p. 71-93, http://dx.doi.org/ 10.1016/j.precamres.2012.09.014

Wang, J. Y., Huang, S. P., and Chen, M. X., 1996, Terrestrial heat flux map, in Yuan, X. C., editor, Geophysical Atlas in China: Beijing, Geophysical Publishing House, 102 p. (in Chinese).

Wang, M., Luo, J. L., Li, M., Bai, X. J., Cheng, C., and Yan, L. W., 2012, Provenance and tectonic setting of sandstone-type uranium deposit in Dongsheng area, Ordos basin: evidence from U-Pb age and Hf isotopes of detrital zircons: Acta Petrologica Sinica.

Wilde, S. A., Zhao, G. C., and Sun, M., 2002, Development of the North China Craton during the Late Archaean and its final amalgamation at $1.8 \mathrm{Ga}$ : Some speculation on its position within a global Palaeoproterozoic Supercontinent: Gondwana Research, v. 5, n. 1, p. 85-94, http://dx.doi.org/10.1016/ S1342-937X (05) 70892-3

Wilde, S. A., Cawood, P. A., Wang, K. Y., Nemchin, A. A., and Zhao, G. C., 2004, Determining Precambrian crustal evolution in China: a case-study from Wutaishan, Shanxi Province, demonstrating the application of precise SHRIMP U-Pb geochronology: Geological Society, London, Special Publications, v. 226, p. 5-25, http://dx.doi.org/10.1144/GSL.SP.2004.226.01.02

Williams, I. S., 1998, U-Th-Pb geochronology by ion microprobe, in McKibben, M. A., Shanks, W. C., III, and Ridley, W. I., editors, Applications of Microanalytical Techniques to Understanding Mineralizing Processes: Reviews in Economic Geology, v. 7, p. 1-35.

Wu, C. H., and Zhong, C. T., 1998, The Paleoproterozoic SW-NE collision model for the central North China Craton: Progress in Precambrian Research, v. 21, p. 28-50 (in Chinese).

Wu, C. H., Sun, M., Li, H. M., Zhao, G. C., and Xia, X. P., 2006, LA-ICP-MS U-Pb zircon ages of the khondalites from the Wulashan and Jining high-grade terrain in northern margin of the North China Craton: constraints on sedimentary age of the khondalite: Acta Petrologica Sinica, v. 22, n. 11, p. 2639-2654 (in Chinese with English abstract).

Wu, J. S., Geng, Y. S., Shen, Q. H., Wan, Y. S., Liu, D. Y., and Song, B., 1998, Archaean Geology Characteristics 
and Tectonic Evolution of China-Korea Paleo-continent: Beijing, Geological Publishing House, 212 p. (in Chinese with English abstract).

Xia, X. P., Sun, M., Zhao, G. C., Wu, F. Y., Xu, P., Zhang, J. H., and Luo, Y., 2006, U-Pb and Hf isotopic study of detrital zircons from the Wulashan khondalites: Constraints on the evolution of the Ordos Terrane, Western Block of the North China Craton: Earth and Planetary Science Letters, v. 241, n. 3-4, p. 581-593, http://dx.doi.org/10.1016/j.epsl.2005.11.024

Xia, X. P., Sun, M., Zhao, G. C., Wu, F. Y., Xu, P., Zhang, J., and He, Y., 2008, Paleoproterozoic crustal growth in the Western Block of the North China Craton: Evidence from detrital zircon Hf and whole rock $\mathrm{Sr}-\mathrm{Nd}$ isotopic compositions of the khondalites in the Jining Complex: American Journal of Science, v. 308, n. 3, p. 304-327, http://dx.doi.org/10.2475/03.2008.05

Xia, X. P., Sun, M., Zhao, G. C., Wu, F. Y., and Xie, L., 2009, U-Pb age and Hf isotopic study detrital zircons from the Lüliang khondalite, North China Craton, and their tectonic significance: Geological Magazine, v. 146, n. 5, p. 701-716, http://dx.doi.org/10.1017/S0016756809006396

Xie, X. Y., and Heller, P. L., 2013, U-Pb detrital zircon geochronology and its implications: the early Late Triassic Yanchang Formation, south Ordos basin, China: Journal of Asian Earth Sciences, v. 64, p. 86-98, http://dx.doi.org/10.1016/j.jseaes.2012.11.045

Yang, Z. S., Li, S. G.,Yu, B. X., Gao, D. H., and Gao, C. Y., 1988, Structural deformation and mineralization in the Early Proterozoic Liaojitite rock suite, eastern Liaoning Province, China: Precambrian Research, v. 39, n. 1-2, p. 31-38, http://dx.doi.org/10.1016/0301-9268(88)90048-4

Yang, Z. S., Xu, Z. Y., and Liu, Z. H., 2000, Khondalite event and Archaean crust structure evolution: Progress in Precambrian Research, v. 23, p. 206-211 (in Chinese with English abstract).

Yin, C. Q., Zhao, G. C., Sun, M., Xia, X. P., Wei, C. J., Zhou, X. W., and Leung, W. H., 2009, LA-ICP-MS U-Pb zircon ages of the Qianlishan Complex: constrains on the evolution of the Khondalite Belt in the Western Block of the North China Craton: Precambrian Research, v. 174, n. 1-2, p. 78-94, http:// dx.doi.org/10.1016/j.precamres.2009.06.008

Zhai, M. G., and Liu, W. J., 2003, Paleoproterozoic tectonic history of the North China craton: a review: Precambrian Research, v. 122, n. 1-4, p. 183-199, http://dx.doi.org/10.1016/S0301-9268(02)00211-5

Zhai, M. G., and Santosh, M., 2011, The early Precambrian odyssey of the North China Craton: A synoptic overview: Gondwana Research, v. 20, n. 1, p. 6-25, http://dx.doi.org/10.1016/j.gr.2011.02.005

Zhai, M. G., Bian, A. G., and Zhao, T. P., 2000, The amalgamation of the supercontinent of North China Craton at the end of Neo-Archaean and its breakup during late Palaeoproterozoic and Mesoproterozoic: Science in China (Series D), v. 43, n. S1, p. 219-232

Zhai, M. G., Guo, J. H., and Liu, W. J., 2005, Neoarchean to Paleoproterozoic continental evolution and tectonic history of the North China craton: Journal of Asian Earth Sciences, v. 24, n. 5, p. 547-561, http://dx.doi.org/10.1016/j.jseaes.2004.01.018

Zhang, C., Han, B. F., Liu, S. W., Ji, J. Q., Zhao, L., and Zhang, L., 2009, SHRIMP U-Pb dating of biotite granites in Daqingshan, Inner Mongolia, and its significance: Acta Petrologica Sinica, v. 25, n. 3, p. 561-567.

Zhang, F. Q., Liu, J. Z., and Ouyang, Z. Y., 1998, Tectonic framework of greenstones in the basement of the North China Craton: Acta Geophysica Sinica, v. 41, p. 99-107.

Zhang, H. F., Zhu, R. X., Santosh, M., Ying, J. F., Su, B. X., and Hu, Y., 2013, Episodic widespread magma underplating beneath the North China Craton in the Phanerozoic: Implications for craton destruction: Gondwana Research, v. 23, n. 1, p. 95-107, http://dx.doi.org/10.1016/j.gr.2011.12.006

Zhao, G. C., and Zhai, M. G., 2013, Lithotectonic elements of Precambrian basement in the North China Craton: Review and tectonic implications: Gondwana Research v. 23, n. 4, p. 1207-1240, http:// dx.doi.org/10.1016/j.gr.2012.08.016

Zhao, G. C., Wilde, S. A., Cawood, P. A., and Lu, L. Z., 1998, Thermal evolution of the Archaean basement rocks from the eastern part of the North China Craton and its bearing on tectonic setting: International Geological Review, v. 40, n. 8, p. 706-721, http://dx.doi.org/10.1080/00206819809465233

$1999 \mathrm{~b}$, Thermal evolution of two textural types of mafic granulites from the North China craton: evidence for both mantle plume and collisional tectonics: Geological Magazine, v. 136, n. 3, p. 223-240, http://dx.doi.org/10.1017/S001675689900254X

Zhao, G. C., Cawood, P. A., and Lu, L. Z., 1999a, Petrology and P-T history of the Wutai amphibolites: implications for tectonic evolution of the Wutai Complex, China: Precambrian Research, v. 93, n. 2-3, p. 181-199, http://dx.doi.org/10.1016/S0301-9268(98)00090-4

Zhao, G. C., Cawood, P. A., Wilde, S. A., Sun, M., and Lu, L. Z., 2000, Metamorphism of basement rocks in the central zone of the North China Craton: implications for Paleoproterozoic tectonic evolution: Precambrian Research, v. 103, n. 1-2, p. 55-88, http://dx.doi.org/10.1016/S0301-9268(00)00076-0

Zhao, G. C., Wilde, S. A., Cawood, P. A., and Sun, M., 2001, Archean blocks and their boundaries in the North China Craton: lithological, geochemical, structural and $P-T$ path constraints and tectonic evolution: Precambrian Research, v. 107, n. 1-2, p. 45-73, http://dx.doi.org/10.1016/S03019268(00) 00154-6

Zhao, G. C., Sun, M., Wilde, S. A., and Li, S. Z., 2005, Late Archean to Paleoproterozoic evolution of the North China Craton: key issues revisited: Precambrian Research, v. 136, n. 2, p. 177-202, http:// dx.doi.org/10.1016/j.precamres.2004.10.002

Zhao, G. C., Wilde, S. A., Sun M., Li, S. Z., Li, X. P., and Zhang, J., 2008a, SHRIMP U-Pb zircon ages of granitoid rocks in the Lüliang complex: Implications for the accretion and evolution of the Trans-North China orogen: Precambrian Research, v. 160, n. 3-4, p. 213-226, http://dx.doi.org/10.1016/ j.precamres.2007.07.004

Zhao, G. C., Wilde, S. A., Guo, J. H., Cawood, P. A., Sun, M., and Li, X. P., 2010, Single zircon grains record two Paleoproterozoic collisional events in the North China Craton: Precambrian Research, v. 177, n. 3-4, p. 266-276, http://dx.doi.org/10.1016/j.precamres.2009.12.007 
Zheng, Y. F., Xiao, W. J., and Zhao, G. C., 2013, Introduction to tectonics of China: Gondwana Research, v. 23, n. 4, p. 1189-1206, http://dx.doi.org/10.1016/j.gr.2012.10.001

Zhong, C. T., Deng, J. F., Wan, Y. S., Mao, D. B., and Li, H. M., 2007, Magma recording of Paleoproterozoic orogeny in central segment of northern margin of North China Craton: Geochemical characteristics and zircon SHRIMP dating of S-type granitoids: Geochimica, v. 36, p. 633-637 (in Chinese with English abstract).

Zhou, X. W., and Geng, Y. S., 2009, Metamorphic age of the khondalite series in the Helanshan region: Constraints on the evolution of the western block in the North China Craton: Acta Petrologica Sinica, v. 25, n. 8, p. 1843-1852 (in Chinese with English abstract). 